\title{
Resonances and particle stochastization in nonhomogeneous electromagnetic fields.
}

\author{
D.L.Vainchtein ${ }^{1,2)}$, E.V.Rovinsky ${ }^{2)}$, L.M.Zelenyi ${ }^{2)}$, and A.I.Neishtadt ${ }^{2}$ ) \\ 1) Department of Mechanical and Environmental Engineering \\ University of California Santa Barbara, CA 93106, USA
}

2) Space Research Institute, ul.Profsouznaya, 84/32, Moscow, Russia, GSP-7, 117997

\begin{abstract}
In the present paper we investigate the resonant interaction between monochromatic electromagnetic waves and charged particles in configurations with magnetic field reversals (e.g. in the earth magnetotail). The smallness of certain physical parameters allows us to solve this problem using perturbation theory reducing the problem of resonant wave-particle interaction to the analysis of slow passages of a particle through a resonance. We discuss in details two of the most important resonant phenomena: capture into resonance and scattering on resonance. We show that these processes result in destruction of the adiabatic invariants, chaotization of particles, may lead to significant (almost free) acceleration of particles and govern transport in the phase space. We calculate the characteristic times of mixing due to resonant effects and separatrix crossings and discuss the relative importance of these phenomena.
\end{abstract}




\section{Introduction.}

In recent years, reduced-order models became a thriving topic of investigation since they allow to observe developing and interaction of coherent structures of different types in both numerical simulations and analytical studies. In most cases even reduced-order dynamical systems contain too many degrees of freedom to allow for analytical solution. Therefore, to obtain an analytical description one must either simplify a model even further risking to lose physical relevance or implement methods from the theory of dynamical systems to obtain approximate solution. One of such approaches is to reduce the coupling between different parts of the system to resonant interactions.

Resonant phenomena occur in a variety of different systems: energy exchange between coupled oscillators, (Gendelman et al., 2001; Vakakis and Gendelman, 2001; Popov at al, 2001), mixing in fluids (Neishtadt et al., 1998; Ward and Homsy, 2001), celestial mechanics and astrophysics (Fux, 2001), billiards (Itin et al, 2001), Josephson junctions (Vasiliev et al., 1997) to name a few recent papers. In the present paper we apply the theory of resonant processes in Hamiltonian systems with slow and fast motions in the form, developed in (Neishtadt, 1999) (see also (Neishtadt, 1997) for references on preceding works) to the analysis of the dynamics of charged particles in nonhomogeneous electromagnetic fields in the magnetotail of the earth.

Magnetospheric tail comes as a product of interaction between the solar wind and the magnetic field of the earth. Solar wind compresses the magnetic field lines on the day side and extends them on the night side of the earth for a few hundred earth radii. Such a structure is a vast reservoir of energy initially stored in magnetic field configuration. This explains tremendous practical interest in understanding the physical processes governing its storage/release cycle, controlling auroral emissions, radio communications and radiation hazards. At the same time the magnetotail is very interesting as a large laboratory for exploration of hot collisionless plasma whose quite a complex behavior can be at least partially deduced from simple mathematical models (Büchner and Zelenyi, 1989; Ashour-Abdalla et al., 1995; Lui, 2001). Magnetic field there is rather weak and trajectories of ions could not be described by the guiding center approximation. The equations of particles' motion are nonintegrable even for the simplest magnetotail geometry and the phase space is partitioned to regions with regular, weakly and strongly chaotic dynamics ((Chen and Palmadesso, 1986); see also (Chen, 1992) for a review). Many features of the ion distributions predicted by these models have been observed in the experimental data (Chen et al., 1990; Ashour-Abdalla et al., 1996), where it was shown that processes in the far region of the magnetotail have a crucial impact on the structure of distribution function of the ions when they approach the earth. 
One of the open problems in this field is the persistence of solutions under various perturbations of the electromagnetic field. Numerical simulations and experimental data show that parts of the initial structure of distribution functions from the far region of the magnetotail survive even when particles come closer to the Earth, while certain features are washed out by the perturbations (see, e.g. (Ashour-Abdalla et al., 1991; AshourAbdalla et al., 1996)), thus further spurring the interest in chaotization and mixing processes in magnetotail. Natural source of perturbations in the magnetotail are the electromagnetic waves (Cattell et al., 1995; Ma and Summers, 1998). The problem of particle's motion in the field of electromagnetic or electrostatic waves is one of the classical problems of plasma physics (Sagdeev, 1983) and was well explored in the case of homogeneous external magnetic field (Karney, et al., 1989; Zaslavskii et al., 1989; Neishtadt et al., 1989; Chernikov et al., 1992; Itin et al., 2000).

In the present paper we study the interaction between particles and a single low-frequency planar monochromatic, electromagnetic or electrostatic, wave in a strongly nonhomogeneous background magnetic field of the magnetotail. The smallness of certain physical parameters allows us to introduce the hierarchical scaling of the slow and fast motions and reduces the problem of wave-particle interaction to the analysis of slow passages of a particle through a nonlinear resonance. Far from the resonances dynamics of particles can be accurately described using the averaging technique. The integrals of motion of the averaged system, obtained in the process of averaging, are adiabatic invariants of the exact system (see e.g. (Arnold et al., 1988)). The scattering on resonance and the capture into resonance lead to destruction of adiabatic invariancy thus controlling the enhanced particles' chaotization and mixing in the phase space.

In our analysis of resonance phenomena we relayed heavily on the theory developed in (Neishtadt, 1997; Neishtadt, 1999). Our aim was neither to generalize nor to improve this theory, but to expand the applicability domain of it (in particular, to illustrate that it can be used when preliminary averaging is required) and to use it to solve a concrete problem of dynamics of charged particles in the earth magnetotail. In the latter part, our main objectives are twofold. First, we want to describe the impact of electromagnetic waves on the particles' dynamics, in particular, to estimate the rate of particle's acceleration. Our second goal is to define the range of parameters of the waves for which the resonance phenomena (and not the separatrix crossings) play the leading role in the stochastization of particles and mixing in the phase space. To achieve this we estimate the rates of mixing due to both processes. As the particles spend only a finite time in the magnetotail, the relation between the characteristic times of mixing and the lifetime of particles in magnetotail determines the extend of mixing due to the respective phenomenon and we compare the characteristic times to describe the relative importance 
of the two phenomena.

The structure of the paper is as follows. In Sections 2 to 5 we discuss the dynamics of charged particles in the field of one electromagnetic wave. In Sect. 2 we introduce the basic equations and approximations for the case of electromagnetic wave. In Sect. 3 we discuss the averaging technique and the motion of particles far from a resonance including jumps of an adiabatic invariant on a separatrix. In Sect. 4 we consider the structure of a resonance in the phase space and discuss the breakdown of the method of averaging. Section 5 contains a detailed description of the general properties of the particles' dynamics in the vicinity of a resonance. Scattering on resonance and the capture into resonance are discussed in Subsect. 5.1 and Subsect. 5.2, respectively. We show that both capture and scattering destroy adiabatic invariancy and we obtain expressions for jumps of adiabatic invariants during a single passage. As the magnitude of the jumps is very sensitive to initial conditions, in the case of multiple passages through resonance these phenomena can be treated as random processes and we derive their statistical properties. In Sect. 6 we discuss the same processes for the case of an electrostatic wave stressing the differences between the influences of the two types of waves. In Sect. 7 we investigate the long-term dynamics of particles, discuss the relative importance of two different chaotization mechanisms and introduce a parameter that characterizes a measure of mixing in the phase space. Section 8 contains the conclusions.

\section{The model of far magnetotail and main equations.}

The earth magnetotail is a product of interaction between the supersonic stream of solar corona particles (solar wind) and the magnetic field of the earth. Solar wind compresses the magnetic field lines on the day side and extends them on the night side of the earth for a few hundreds earth radii. Charged particles from the solar wind enter the magnetotail in the far region and then accelerate and drift towards the earth. In the equatorial plane of the magnetospheric tail there is the current sheet, which supports antiparallel magnetic fields in northern/southern lobes of the tail (Fig. 1a). The region between the earth at the left and the so-called $X$-line at the right of Fig. 1a, is called "field reversal region." Magnetic field there is rather weak and trajectories of ions could not be described by well known guiding center approximation.

One of the simplest approximations of the magnetic field, $\mathbf{B}$, which is adequate to incorporate main physical effects is the so-called parabolic model (see e.g. Chen, 1992) (Fig. 1b):

$$
\mathbf{B}=B_{0} \frac{z}{L} \mathbf{e}_{x}+B_{n} \mathbf{e}_{z} .
$$

Here $\mathbf{e}_{i}$ are the unit vectors of a Cartesian coordinate system in which the $x$-axis is directed towards the earth, 
(a)
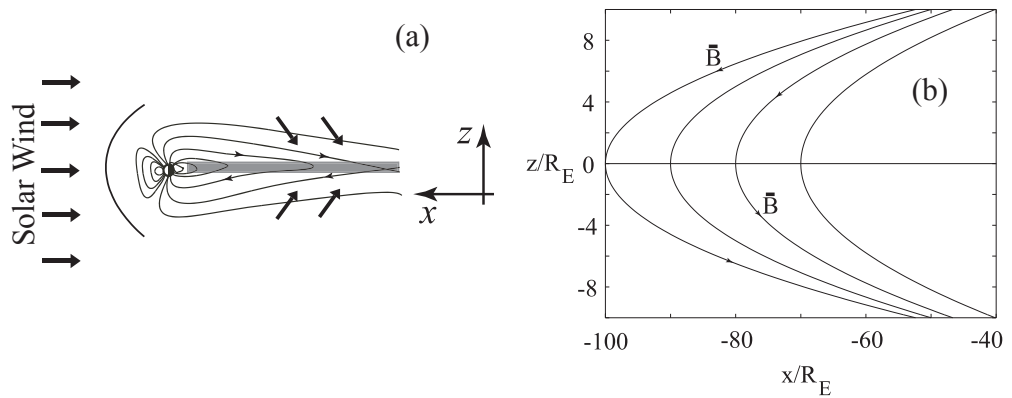

Figure 1: Configuration of the earth magnetotail. (a) General view (not to scale), the shaded area in the center is the current sheet. (b) Field lines of the magnetic field in the parabolic model: at every point magnetic field is tangential to the field line and the field strength is proportional to the density of the field lines. $R_{E}$ is the radius of the earth.

the $y$-axis is in the equatorial plane and is directed from dusk to dawn (perpendicular to the plane of view in Fig. 1a) and the $z$-axis is perpendicular to the equatorial plane and is directed from south to north (see Fig. 1a); $L$ is the characteristic transverse length scale. $B_{n}$ and $B_{0}$ are constants.

The impact of variations of $B_{n}$ as a function of $x$ on the dynamics of particles was studied in (Zelenyi et al., 1990; Karimabadi et al., 1990; Vainshtein et al., 1995). It was shown that variations of $B_{n}$ together with the presence of constant electric field (in magnetotail this electric field is induced by the solar wind, $\mathbf{E}=E_{c} \mathbf{e}_{y}$ ) lead to the acceleration of particles, thus limiting the time particles spend in the magnetotail (see Sect. 7). Otherwise, the dynamics is quite similar with and without the constant electric field. If $B_{n}=$ const, we can exclude $E_{c}$ from the Hamiltonian of a charged particle by switching to a moving coordinate frame $x^{\prime}=x-t c E_{c} / B_{n}$ (de Hoffman-Teller substitution, (de Hoffman and Teller, 1950)). Therefore, in what follows we do not take $E_{c}$ into account, assuming that the de Hoffman-Teller substitution was made.

What was ignored in the previous studies is the influence of time-dependent perturbations of electromagnetic fields. One of the most natural ways to consider these perturbations is to include low-frequency electromagnetic or electrostatic waves. In the present paper, we discuss the influence of either one monochromatic electromagnetic wave with the vector of the electric field directed along the $y$-axis or one electrostatic wave with the wave vector residing in the equatorial plane. The impact of two types of waves on the particle's dynamics turns out to be quite similar. We start with one electromagnetic wave and return to an electrostatic wave in Section 6 .

The vector potential of a charged particle in the field of an electromagnetic wave is

$$
\mathbf{A}=\left(0 ; B_{n} x-B_{0} \frac{z^{2}}{2 L}-\frac{c E}{\bar{\omega}} \sin (\overline{\mathbf{k}} \mathbf{x}-\bar{\omega} t) ; 0\right)
$$

where $\overline{\mathbf{k}}$ and $\bar{\omega}$ are the wave vector and the frequency of the wave, respectively, $t$ is the time, and $c$ is the speed 
of light. This vector potential corresponds to the following configuration of electromagnetic field:

$$
\begin{aligned}
& \mathbf{B}=\operatorname{rot} \mathbf{A}=\left(B_{0} \frac{z}{L}+\frac{c E \bar{k}_{z}}{\bar{\omega}} \cos (\overline{\mathbf{k}} \mathbf{x}-\bar{\omega} t)\right) \mathbf{e}_{x}+\left(B_{n}-\frac{c E \bar{k}_{x}}{\bar{\omega}} \cos (\overline{\mathbf{k}} \mathbf{x}-\bar{\omega} t)\right) \mathbf{e}_{z}, \\
& \mathbf{E}=-\frac{1}{c} \frac{\partial \mathbf{A}}{\partial t}=-E \cos (\overline{\mathbf{k}} \mathbf{x}-\bar{\omega} t) \mathbf{e}_{y} .
\end{aligned}
$$

The Hamiltonian of a charged particle has the form

$$
H=\frac{1}{2 m}\left(\mathbf{P}-\frac{e}{c} \mathbf{A}\right)^{2}=\frac{1}{2 m}\left\{P_{x}^{2}+\left[P_{y}-\frac{e}{c}\left(B_{n} x-B_{0} \frac{z^{2}}{2 L}-\frac{c E}{\bar{\omega}} \sin (\overline{\mathbf{k}} \mathbf{x}-\bar{\omega} t)\right)\right]^{2}+P_{z}^{2}\right\}
$$

where $\mathbf{P}=\left(P_{x}, P_{y}, P_{z}\right), m$ and $e$ are the generalized momentum, the mass and the charge of a particle, respectively. We introduce a set of non-dimensional variables and parameters

$$
\begin{aligned}
& \mathbf{x}_{1}=\mathbf{x} / \sqrt{\rho L}, \quad h_{1}=H / m v^{2}, \quad \mathbf{k}=\overline{\mathbf{k}} \sqrt{\rho L}, \quad \kappa=\frac{B_{n}}{B_{0}} \sqrt{L / \rho}, \\
& \mathbf{P}_{1}=\mathbf{P} / m v, \quad t_{1}=t \frac{v}{\sqrt{\rho L}}, \quad \omega=\bar{\omega} \frac{\sqrt{\rho L}}{v}, \quad \varepsilon=c E / v B_{n},
\end{aligned}
$$

where $v$ is a typical velocity of a particle and $\rho=\mathrm{cmv} /\left(e B_{0}\right)$ is a characteristic Larmor radius in asymptotic field $B_{0}$. We restrict our consideration to the case when the wave vector resides in the equatorial plane: $\mathbf{k}=\left(k_{x}, k_{y}, 0\right)$. To be definite, we assume $k_{x}, k_{y} \geq 0$ and let $\omega$ be of any sign to account for the waves that move in either direction along the $x$-axis (or the $y$-axis). The other cases $\left(k_{x}<0\right.$ or $\left.k_{y}<0\right)$ can be considered in the same manner.

The dimensionless Hamiltonian of a charged particle is

$$
h_{1}=\frac{1}{2}\left\{P_{x_{1}}^{2}+P_{z_{1}}^{2}+\left[P_{y_{1}}-\kappa\left(x_{1}-\frac{\varepsilon}{\omega} \sin \left(k_{x} x_{1}+k_{y} y_{1}-\omega t_{1}\right)\right)+\frac{1}{2} z_{1}^{2}\right]^{2}\right\} .
$$

Apply change of variables with a generating function

$$
S=P_{\varphi} \frac{1}{k}\left(k_{x} x_{1}+k_{y} y_{1}-\omega t_{1}\right)+P_{x_{2}} x_{1}+P_{z_{2}} z_{1}, \quad k=|\mathbf{k}|,
$$

that corresponds to the introduction of a phase of the wave, $\varphi$ :

$$
\begin{array}{ccc}
\varphi=\frac{1}{k}\left(k_{x} x_{1}+k_{y} y_{1}-\omega t_{1}\right), & x_{2}=x_{1}, & z_{2}=z_{1}, \\
P_{\varphi}=\frac{k}{k_{y}} P_{y_{1}}, & P_{x_{2}}=P_{x_{1}}-\frac{k_{x}}{k} P_{\varphi}, & P_{z_{2}}=P_{z_{1}} .
\end{array}
$$

The new Hamiltonian is

$$
h_{2}=h_{1}-P_{\varphi} \frac{\omega}{k}
$$


and in the new variables takes the form (we do not write the subscripts 1 and 2)

$$
h=\frac{1}{2}\left[\left(P_{x}+\frac{k_{x}}{k} P_{\varphi}\right)^{2}-2 \frac{\omega}{k} P_{\varphi}+P_{z}^{2}+\left(\frac{k_{y}}{k} P_{\varphi}-\kappa x+\frac{1}{2} z^{2}+\kappa \frac{\varepsilon}{\omega} \sin (k \varphi)\right)^{2}\right] .
$$

The new "energy", $h$ (that differs from the usual energy by a quantity $\frac{\omega}{k} P_{\varphi}$ ), is an integral of motion.

Now we must make some approximations. Parameter $\kappa$ can be written as $\kappa=\sqrt{\rho_{c} / \rho}$, where is $\rho_{c}$ is minimal curvature radius of a magnetic field line (which is achieved at $z=0$ ). If $\kappa \gg 1$, particles are magnetized and their motion can be described by the guiding center theory (see e.g (Chen, 1992)). In the magnetotail electrons are always magnetized, except for the very close vicinity of the $X$-line. The value of $\kappa$ for ions depends on the distance from the earth and can be anywhere between 0.05 in the far magnetotail and 3 close to the earth. We are interested in the case $\kappa \ll 1$, which is realized for ions beyond $20 R_{E}$, where $R_{E}$ is the radius of the earth. In this case the typical size of the system in the $x$-direction $(\sim L / \kappa)$ is much larger than the typical size of the system in the $z$-direction $(\sim L)$ and also

$$
\omega_{x} \ll \omega_{z}
$$

where $\omega_{z}$ and $\omega_{x}$ are the typical frequencies of motion in $z$-and $x$-directions, respectively.

For a variety of electromagnetic waves present in the magnetotail the wavelength $(\sim 1 / k)$ is much smaller than the typical size of the system in the $x$-direction and much larger than the typical size of the system in the $z$-direction and the frequency residing between $\omega_{z}$ and $\omega_{x}$. Taking normalizing conditions (1) into account and assuming that $v \sim v_{A} \sim v_{T i}$, where $v_{A}$ is the Alfven velocity and $v_{T i}$ is the thermal velocity of ions, we arrive at the following inequalities:

$$
\kappa \ll \omega \ll 1, \quad \kappa \ll k \sqrt{\rho / L} \ll 1
$$

The above inequalities allow us to introduce a hierarchy of motions and to solve the problem using the perturbation theory.

\section{The averaged system.}

Hamiltonian (3) possesses three degrees of freedom. Under the above assumptions on the values of the parameters of the problem, we can introduce the hierarchy of time scales. The fastest motion is $z$-motion, the second fastest is the time scale associated with the wave and the slowest is $x$-motion. In the present section we apply two successive averagings (the first over $z$-motion and the second over the period of the wave) to describe the long-term dynamics of charged particles. 

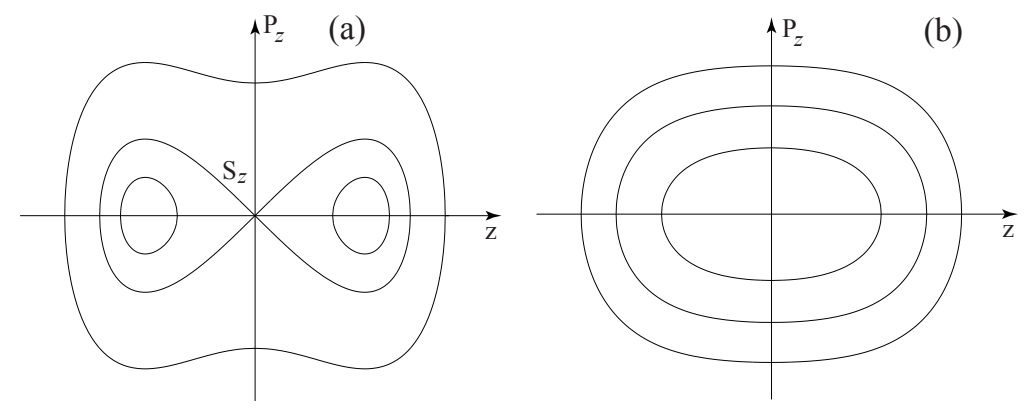

Figure 2: Characteristic phase portrait of $z$-motion for (a) $\zeta>0$ and (b) $\zeta<0$.

The properties of $z$-motion, governed by the Hamiltonian

$$
h_{z}=\frac{1}{2}\left[P_{z}^{2}+\left(\zeta-\frac{z^{2}}{2}\right)^{2}\right],
$$

where $\zeta=-\left(k_{y} / k\right) P_{\varphi}+\kappa x-\kappa(\varepsilon / \omega) \sin (k \varphi)$, were considered in (Zelenyi et al., 1990).

When studying the $z$-motion, we fix the values of $x, P_{x}, \varphi$ and $P_{\varphi}$. Characteristic phase portraits of $z$-motion are schematically shown in Fig. 2a for $\zeta>0$ and in Fig. 2b for $\zeta<0$. The separatrix $S_{z}$ in Fig. 2a is specified by $h_{z}=\zeta^{2} / 2$. The action variable,

$$
I_{z}=\frac{1}{2 \pi} \oint P_{z} d z
$$

is equal to the normalized by $2 \pi$ area inside a phase curve on the $\left(z, P_{z}\right)$ plane. Upon averaging equation of motion over z-motion (cf. (Büchner and Zelenyi, 1989)) we get

$$
h=\frac{1}{2}\left(P_{x}+\frac{k_{x}}{k} P_{\varphi}\right)^{2}-\frac{\omega}{k} P_{\varphi}+h_{z}\left(\zeta, I_{z}\right),
$$

where

$$
\delta=\varepsilon / \omega
$$

and $h_{z}(\cdot)$ is the Hamiltonian of $z$-motion, expressed in terms of slow variables and $I_{z}$, that is an adiabatic invariant of exact system (3) (see (Büchner and Zelenyi, 1989) for the explicit form of $h_{z}$. The effects arising from the dependence of $h_{z}$ on $I_{z}$ were studied before (see (Büchner and Zelenyi, 1989; Vainshtein et al., 1995)). It was shown that $h_{z}$ satisfies a scaling law: $h_{z}\left(\zeta, I_{z}\right)=I_{z}^{-4 / 3} h_{z}\left(\zeta I_{z}^{-2 / 3}, 1\right)$, and in what follows we omit $I_{z}$ from the arguments of $h_{z}$. Figure 3 presents the profiles of $h_{z}(\zeta)$ and its derivative with respect to the first argument, $h_{z}^{\prime}(\zeta)$, for $I_{z}=1$ (cf. (Büchner, 1986)). In Fig. 3, $h_{z}^{\prime}(\zeta)$ has a discontinuous derivative at $\zeta_{s}=(3 \pi / 8)^{2 / 3}$ and $\zeta_{m} \approx 0.59 \zeta_{s}$ is the zero of $h_{z}(\zeta)$. 


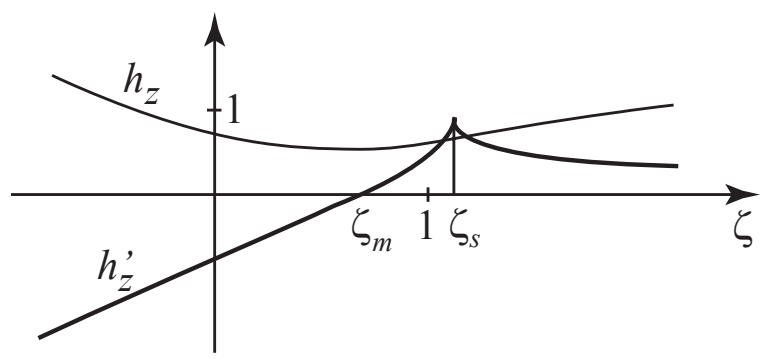

Figure 3: Profiles of the functions $h_{z}(\zeta)$ and $h_{z}^{\prime}(\zeta)$ for $I_{z}=1$.

To consider a wave as a perturbation, we must assume that the amplitude of the wave is small:

$$
\kappa \delta \ll 1
$$

Using (1), this condition can be rewritten in dimensional form as

$$
e E / m v \bar{\omega} \ll 1 .
$$

Note, that $\bar{\omega}$ is dimensional frequency of the wave (see (1)). The physical meaning of the above inequality is that the maximum momentum that a particle could gain in one period of the wave is much smaller then the initial momentum. If the above condition is satisfied, the term $\kappa \delta \sin (k \varphi)$ in the argument of $h_{z}$ is small and $h_{z}$ can be approximated by the first two terms of the series expansion:

$$
\begin{aligned}
h & =\frac{1}{2}\left(P_{x}+\frac{k_{x}}{k} P_{\varphi}\right)^{2}-\frac{\omega}{k} P_{\varphi}+h_{z}\left(-\frac{k_{y}}{k} P_{\varphi}+\kappa x\right)-\kappa \delta h_{z}^{\prime} \sin (k \varphi) \\
& =h_{a v}\left(\kappa x, P_{x}, P_{\varphi}\right)-\kappa \delta h_{z}^{\prime} \sin (k \varphi) .
\end{aligned}
$$

In (5), $h_{a v}$ is the average value of $h$ over $\varphi$.

Hamiltonian (5) possesses two degrees of freedom, of which the variable $\varphi$ is fast and the variables $x, P_{x}, P_{\varphi}$ are slow. Therefore, in the next approximation we can average the motion over fast $\varphi$-oscillations. This approximation is valid everywhere except for a small part of the phase space where $\dot{\varphi} \approx 0$. This (second overall) averaging of (5) over $\varphi$ corresponds to omitting the term $\kappa \delta h_{z}^{\prime} \sin (k \varphi)$ and reduces the Hamiltonian $h$ to $h_{a v}$ :

$$
h=h_{a v}=\frac{1}{2}\left(P_{x}+\frac{k_{x}}{k} P_{\varphi}\right)^{2}-\frac{\omega}{k} P_{\varphi}+h_{z}\left(-\frac{k_{y}}{k} P_{\varphi}+\kappa x\right) .
$$

As a result, $P_{\varphi}$ becomes an integral of motion and the problem reduces to the one already solved (cf. (Büchner and Zelenyi, 1989; Zelenyi et al., 1990)): the averaging is equivalent to neglecting the wave. Therefore, it follows 


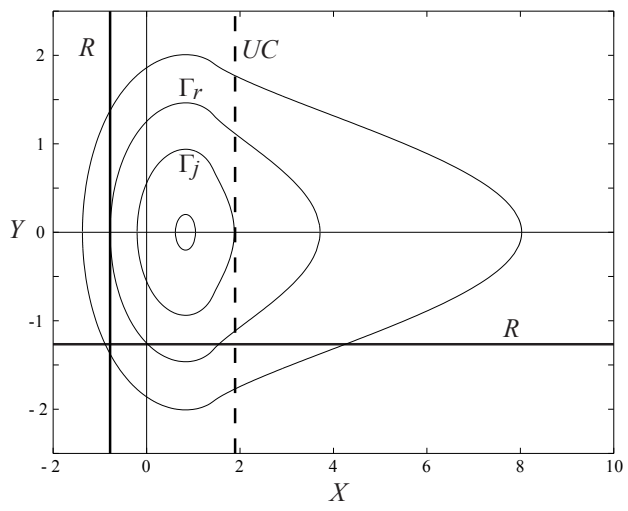

Figure 4: The phase portrait of the system with the Hamiltonian $h_{a v}$ on the $(X, Y)$ plane (see (7)). On the whole phase plane $P_{\varphi}=P_{\varphi, 0}$. The horizontal and vertical solid lines are the intersections of $P_{\varphi}=P_{\varphi, 0}$ plane and the resonant surface for $k_{y}=0$ and $k_{x}=0$, respectively.

from (2), that in the double-averaged system the energy of particles does not change. The quantity $P_{\varphi}$ is the adiabatic invariant of the exact system: in the absent of resonances it would be conserved with the accuracy of order $\kappa \delta$ over times of order $1 / \kappa \delta$ (see (Bogolyubov and Mitropolsky, 1961)).

Figure 4 presents the phase portrait of the system with the Hamiltonian $h_{a v}$ on the $(X, Y)$ plane, defined by $P_{\varphi}=P_{\varphi, 0}=$ const, $I_{z}=I_{z, 0}=$ const. The new variables, $X$ and $Y$, are given by

$$
X=-\frac{k_{y}}{k} P_{\varphi}+\kappa x, \quad Y=P_{x}+\frac{k_{x}}{k} P_{\varphi} .
$$

Note, that $\zeta=X-\kappa(\varepsilon / \omega) \sin (k \varphi)$, in other words, up to the small (and disappearing upon the averaging) term $X$ is equivalent to $\zeta$. The qualitative structure of the phase portrait is the same for all values of $P_{\varphi}$ and $I_{z}$. For example, there is always only one, elliptic, fixed point. The horizontal and vertical thick lines are the intersections of the phase plane and resonant surfaces for $k_{y}=0$ and $k_{x}=0$, respectively (see Sect. 4 below). The vertical line denoted as $\mathbf{U C}$ is discussed in the following subsection.

\subsection{Separatrix crossings}

The vertical thick dashed line is the uncertainty curve, UC (see (Wisdom, 1985)). This line corresponds to the separatrix of the $z$-motion: when a phase curve of averaged system (6) intersects $\mathbf{U C}$, a phase curve of the $z$-motion resides on the separatrix of the $z$-motion, $S_{z}$, (see (Vainshtein et al., 1995) for the detailed description). To the right of UC, particles move inside of two separatrix loops in Fig. 2a; while to the left of UC, particles encircle the origin in Fig. 2a or Fig. 2b depending on the sign of $\zeta$. It is the presence of $\mathbf{U C}$ that leads to 
discontinuity of $h_{z}^{\prime \prime}$. A special phase curve $\Gamma_{j}$, defined by

$$
2\left(h_{\Gamma_{j}}+\frac{\omega}{k} P_{\varphi}\right)=\left((8 / 3 \pi) I_{z}\right)^{4 / 3}
$$

is tangent to $\mathbf{U C}$. It divides the phase plane into two regions: trajectories residing within $\Gamma_{j}$ do not cross $\mathbf{U C}$, and those residing outside $\Gamma_{j}$ cross $\mathbf{U C}$.

The value of $I_{z}$ is conserved only far from UC and undergoes a jump every time a phase curve crosses UC. The asymptotic formulas for the jump of adiabatic invariant on a separatrix were obtained by Timofeev (Timofeev, 1978) for particular case of a pendulum in a slowly varying gravity field, by Neishtadt (Neishtadt, 1986) and Cary and co-workers (Cary et al., 1986) for systems with one degree of freedom plus slowly varying parameter and by Neishtadt (Neishtadt, 1987) for systems with two degrees of freedom, one corresponding to the fast motion and the other corresponding to the slow motion. This theory was first applied to magnetospheric problems in (Büchner and Zelenyi, 1989). Modifying the corresponding equation from (Büchner and Zelenyi, 1989) to accommodate for the presence of the wave, we have for the leading order approximation the magnitude of the jump

$$
\Delta I_{z}=\mp \frac{4}{\pi} \kappa\left(P_{x}+\frac{k_{x}}{k} P_{\varphi}\right) \ln \left(2 \sin \pi \Theta_{s}\right)
$$

In (8), $\Delta I_{z}$ is the difference between the value of $I_{z}$ outside of $S_{z}$ and twice the value of $I_{z}$ inside $S_{z}$; the minus and plus signs correspond to the crossing of $\mathbf{U C}$ from left to right and from right to left in Fig. 3, respectively. The phase $\Theta_{s} \in(0,1)$ characterizes a separatrix crossing and it depends not only on $x, P_{x}, \varphi$ and $P_{\varphi}$, but on $z$ and $P_{z}$ as well. The error term in (8) is of order $\kappa^{3 / 2} \log \kappa$, provided that $\Theta_{s}$ is at the distance of order 1 from both 0 and 1 . The value of $\Theta_{s}$ is very sensitive to small perturbations of the initial conditions and it is shown in (Neishtadt, 1987), that in the limit $\kappa \rightarrow 0$ it can be treated as a random value uniformly distributed on $(0,1)$ interval. In this case $\Delta I_{z}$ can be also treated as a random value with a zero mean and dispersion of order $\sim \kappa^{2}$. Accumulation of the jumps may lead to chaotization of particles' motion. Because of these jumps, $I_{z}$ was referred to as a quasi-adiabatic invariant in (Büchner and Zelenyi, 1989; Vainshtein et al., 1995).

In the present paper we assume that the intersections of a given trajectory with UC and the areas where $\dot{\varphi} \approx 0$ do not overlap. In this case the influence of separatrix crossings and resonance crossings (see below, Sect. 5) on the dynamics of particles can be studied separately.

Far from the domains where $\dot{\varphi} \approx 0$ the presence of the term $-\kappa \delta h_{z}^{\prime} \sin (k \varphi)$ leads to small oscillations of an exact trajectory around the corresponding averaged one. These oscillations give rise to two phenomena:

- The domain of separatrix crossings is augmented by a strip of the width of order $\kappa \delta \ll 1$ as if an averaged 
trajectory comes within $\kappa \delta$ from the $\mathbf{U C}$, the corresponding exact trajectory may cross UC.

- The exact expression for the magnitude of the jumps of the adiabatic invariant of $z$-motion, $I_{z}$, changes although the statistics of the diffusion of $I_{z}$ remains the same.

\section{Structure of the resonance and breakdown of the method of av- eraging.}

In the present section and in Sect. 5 we apply the general theory of resonance crossings in Hamiltonian systems with slow and fast motions (Neishtadt, 1999) to the problem under consideration. Our aim is to expand the applicability domain of this theory (in particular, to illustrate that it can be used when preliminary averaging is required) and to use it to solve a concrete problem of dynamics of charged particles in the earth magnetotail. It is worth noting that despite double averaging, it is possible to obtain all the estimates explicitly. In particular, although $h_{z}$ is an implicit function of both $\kappa x$ and $P_{\varphi}$ (see (Büchner and Zelenyi, 1989)) the explicit expressions for the parameters controlling the resonance crossings (see Eqns. (13) - (16) below) can be obtained.

The equation $\dot{\varphi}=0$ defines a $2-\mathrm{D}$ surface, in the $3-\mathrm{D}\left(\kappa x, P_{x}, P_{\varphi}\right)$ phase space, that we call the resonant surface, or the resonance, and denote by $R$. At the resonance the projection of the averaged velocity of a particle on the direction of the wave vector is equal to the phase speed of the wave. This phenomena is similar to the classical Cherenkov-type resonance $\omega=(\mathbf{k}, \widehat{\mathbf{v}})$ but $\widehat{\mathbf{v}}$ here stands not for the instant, but the averaged (over fast z-oscillations) velocity of a particle.

In the vicinity of a resonance the variable $\varphi$ is not fast compared with $x$. Hence, we can not expect averaged system (6) to approximate the exact system adequately. As a result, the value of the integral of the averaged system, $P_{\varphi}$, may change in the process of a passing through the vicinity of the resonance. The change in $P_{\varphi}$ leads to the corresponding change in the energy of a particle (see (2)).

The resonance $R$ is given by

$$
\dot{\varphi}=\frac{\partial h_{a v}}{\partial P_{\varphi}}=\left(P_{x}+\frac{k_{x}}{k} P_{\varphi}\right) \frac{k_{x}}{k}-\frac{\omega}{k}-\frac{k_{y}}{k} h_{z}^{\prime}=0 .
$$

The structure of resonances, and dynamics of particles in general, are simpler for particular cases $k_{y}=0$ or $k_{x}=0$.

1. $k_{y}=0$.

The equation defining the resonant surface has the form

$$
-\frac{\omega}{k}+\left(P_{\varphi}+P_{x}\right)=0
$$


In this case $R$ is a plane that is parallel to the $\kappa x$-axis.

2. $k_{x}=0$.

The equation defining the resonant surface has the form

$$
-\frac{\omega}{k}-h_{z}^{\prime}\left(-P_{\varphi}+\kappa x\right)=0
$$

It follows from the profile of $h_{z}^{\prime}$ in Fig. 3, that Eqn. (10) either has no solutions, or defines one or two planes, that are parallel to the $P_{x}$-axis.

The horizontal and vertical thick lines in Fig. 4 are the intersections of the resonant surface and the plane $P_{\varphi}=P_{\varphi, 0}$ for $k_{y}=0$ and $k_{x}=0$, respectively. For every resonance curve there is a special phase trajectory, $\Gamma_{r}$, that is tangent to $R$ (the one shown in Fig. 4 corresponds to $k_{x}=0$ ) and it divides the phase space into two parts: trajectories residing within $\Gamma_{r}$ do not cross the resonance, and those residing outside $\Gamma_{r}$ cross the resonance.

A characteristic trajectory of exact system looks as follows. A particle approaches the resonant zone with the value of $P_{\varphi}$ oscillating with a small amplitude, $\sim(\kappa \delta)$, near some value $P_{\varphi}^{-}$. When in the process of the motion it arrives to the resonant zone is either captured into the resonance, or crosses the resonant zone without being captured. (Actually, there is also some intermediate regime of motion in the resonant zone, but it occurs for a very small measure of initial conditions; we will not discuss it.) Phenomenologically, the difference between the two regimes of motion in the resonant zone can be described as follows. In the case of capture, upon the arrival to the resonant zone the phase, $k \varphi$, switches its behavior from rotation ( $\varphi$ changes monotonically, a trajectory encircle the phase cylinder) to oscillation ( $\varphi$ changes between two values, $\dot{\varphi}$ changes the sign twice during each period). In the case of crossing the resonant zone without capture, $\dot{\varphi}$ changes the sign only once in the resonant zone. The two phenomena have qualitatively different impact on the particle's dynamics. After the passage through the resonant zone (and far from the resonance) the value of $P_{\varphi}$ oscillates near some other value, $P_{\varphi}^{+}$, again with a small amplitude $\sim(\kappa \delta)$.

In the rest of the present section we provide a qualitative description of these phenomena and return to a qualitative description in the next section.

In the case of capture into resonance, upon the arrival to the resonant zone a phase point drifts for a long time (of order $\sim 1 /(\kappa \delta)$ ) along the resonant surface. As a result, $P_{\varphi}$ changes by a value of order 1 . Among all the particles that arrive to the resonant zone during a given time interval of order $\sim 1 /(\kappa \delta)$ only a small part, of order $\sim \sqrt{\kappa \delta}$, is captured. Initial conditions for particles that are or are not captured are mixed. Therefore, 
it is reasonable to consider capture as probabilistic phenomenon. For a particular particle the probability to be captured is small, of order $\sim \sqrt{\kappa \delta}$.

The particles that cross the resonant zone without being captured typically pass through this zone in time of order $\sim 1 / \sqrt{\kappa \delta}$ (see (Neishtadt, 1997; Neishtadt, 1999) for more accurate estimates). The major resonant phenomenon for such particles is the scattering on resonance. In this case the difference, $\Delta P_{\varphi}=P_{\varphi}^{+}-P_{\varphi}^{-}$, that is typically of order $\sim \sqrt{\kappa \delta}$, is referred to as the amplitude of the scattering or, as $P_{\varphi}$ is the adiabatic invariant of the averaged system, as a jump of the adiabatic invariant. This value is very sensitive to small changes of the initial conditions: a change of the initial conditions far from a resonance by a quantity of order $\sim \kappa \delta$ leads to a big (of order $\sim 1$ compared with the typical values) change in the amplitude of the scattering. Hence, the scattering can be considered as a random process.

As the passages through the vicinity of a resonance lead to the destruction of adiabatic invariancy of $P_{\varphi}$, the dynamics of particles becomes chaotic. There is a crucial difference between these resonant phenomena and certain other routes to chaos, like separatrix splitting. Although the total size of the resonant zone is small with the magnitude of the perturbation and it is localized in a small domain in the phase space, the effect is large and global: the chaotic domain is of the order 1 (of the order of the total phase volume of the system) regardless of the magnitude of perturbation.

\section{The description of motion in the vicinity of the resonance.}

In the present section we consider the resonance processes for particles in the field of one electromagnetic wave. A vicinity of the resonant surface, where $|\dot{\varphi}|<\operatorname{const} \sqrt{\kappa \delta}$, is called a resonant zone. Let us derive equations of motion in this zone. Expanding Hamiltonian (5) as a power series over $P_{\varphi}-P_{\varphi, \text { res }}$, where $P_{\varphi, \text { res }}$ is the value of $P_{\varphi}$ on the resonance, and keeping the first three terms we get:

$$
\begin{aligned}
h & \approx h_{r e s}\left(\kappa x, P_{x}\right)+\left.\frac{1}{2} \frac{\partial^{2} h_{a v}}{\partial P_{\varphi}^{2}}\right|_{r e s}\left(P_{\varphi}-P_{\varphi, r e s}\right)^{2}-\kappa \delta h_{z}^{\prime} \sin (k \varphi), \\
h_{r e s}\left(\kappa x, P_{x}\right) & =h_{a v}\left(\kappa x, P_{x}, P_{\varphi, r e s}\right) .
\end{aligned}
$$

In (11), we must express $P_{\varphi, \text { res }}=P_{\varphi, \text { res }}\left(\kappa x, P_{x}\right)$ from (9). The neglected terms in (11) are of order $O(\kappa \delta)^{3 / 2}$. Recall that, by definition of a resonance (see (10)), $\partial h_{a v} / \partial P_{\varphi}$ is zero on $R$. Apply a change of variables with a generating function

$$
S=\left(\bar{P}_{\varphi}+P_{\varphi, r e s}\left(\kappa x, \bar{P}_{x}\right)\right) \varphi+x \bar{P}_{x},
$$


that can be explicitly written as

$$
\begin{array}{rlrl}
\bar{x} & =\frac{\partial S}{\partial \bar{P}_{x}}=x+\frac{\partial P_{\varphi, \text { res }}}{\partial \bar{P}_{x}} \varphi, & \bar{\varphi}=\varphi, & \\
P_{x}=\frac{\partial S}{\partial x}=\bar{P}_{x}+\kappa \frac{\partial P_{\varphi, \text { res }}}{\partial \kappa x} \varphi, & P_{\varphi}=\bar{P}_{\varphi}+P_{\varphi, \text { res }}
\end{array}
$$

Although the value of $\varphi$ changes while a particle moves in the resonant zone, the total change of this value is small compared with $1 / \kappa$. As $\varphi$ is defined up to an additive constant, one can choose this constant to make $\kappa \varphi$ small and, therefore, to make substitution (12) close to identity in the vicinity of $R$. Expanding the first term in Hamiltonian (11) over $\kappa \varphi \ll 1$ and substituting $h_{r e s}$ from (5) into (11) we arrive at

$$
h=h_{x}+\kappa h_{\varphi}
$$

where

$$
\begin{aligned}
h_{x} & =\frac{1}{2}\left(\bar{P}_{x}+\frac{k_{x}}{k} P_{\varphi, r e s}\right)^{2}-\frac{\omega}{k} P_{\varphi, \text { res }}+h_{z}\left(-\frac{k_{y}}{k} P_{\varphi, r e s}+\kappa \bar{x}\right), \\
h_{\varphi} & =\frac{k^{2}}{k_{x}^{2}+k_{y}^{2} h_{z}^{\prime \prime}}\left[\frac{k_{x}}{k} h_{z}^{\prime}+\frac{k_{y}}{k}\left(P_{x}+\frac{k_{x}}{k} P_{\varphi, r e s}\right) h_{z}^{\prime \prime}\right] \varphi \\
& +\frac{1}{2} \frac{1}{\kappa} \frac{k_{x}^{2}+k_{y}^{2} h_{z}^{\prime \prime}}{k^{2}} \bar{P}_{\varphi}^{2}-\delta h_{z}^{\prime} \sin (k \varphi) .
\end{aligned}
$$

In (14) and (15) we must substitute $P_{\varphi, \text { res }}=P_{\varphi, \text { res }}\left(\kappa \bar{x}, \bar{P}_{x}\right)$. Equations (13) - (15) are valid away from the singularity of $h_{z}^{\prime \prime}$ (see Sect. 2). The explicit form of Eqn. (15) is quite remarkable, as $h_{z}$, obtained by the averaging the exact Hamiltonian over $z$-motion, is an implicit function of both $\kappa x$ and $P_{x}$ (see (Büchner and Zelenyi, 1989)). Denote

$$
\begin{aligned}
b & =\frac{k^{2}}{k_{x}^{2}+k_{y}^{2} h_{z}^{\prime \prime}}\left[\frac{k_{x}}{k} h_{z}^{\prime}+\frac{k_{y}}{k}\left(\bar{P}_{x}+\frac{k_{x}}{k} P_{\varphi, \text { res }}\right) h_{z}^{\prime \prime}\right], \\
g & =\frac{k_{x}^{2}+k_{y}^{2} h_{z}^{\prime \prime}}{k^{2}} .
\end{aligned}
$$

In terms of $b$ and $g, h_{\varphi}$ takes the form

$$
\kappa h_{\varphi}=\kappa b \varphi+\frac{1}{2} g \bar{P}_{\varphi}^{2}-\kappa \delta h_{z}^{\prime} \sin (k \varphi) .
$$

Note, that (nearly) all the discussion of the previous and current sections is quite general and is a direct reworking of (Neishtadt, 1999). Particular properties of the problem at hand come to play are in deriving Eqns.(15) - 
(17) and will become important later, when we discuss statistical properties of capture and scattering and the effects of multiple passages through resonance.

In certain particular cases the equations of motion can be simplified:

1. $k_{y}=0$.

$$
\begin{aligned}
\dot{\bar{x}} & =\omega / k \\
\dot{\bar{P}} & =-\kappa h_{z}^{\prime} \\
\kappa h_{\varphi} & =\kappa(\varphi-\delta \sin (k \varphi)) h_{z}^{\prime}+\frac{1}{2} \bar{P}_{\varphi}^{2}
\end{aligned}
$$

2. $k_{x}=0$.

$$
\begin{aligned}
\dot{\bar{x}} & =\bar{P}_{x} \\
\dot{\bar{P}}_{x} & =\kappa \omega / k \\
\kappa h_{\varphi} & =\kappa \bar{P}_{x} \varphi+\kappa \frac{\omega}{k} \delta \sin (k \varphi)+\frac{1}{2} h_{z}^{\prime \prime} \bar{P}_{\varphi}^{2}
\end{aligned}
$$

In the last line we substituted $h_{z}^{\prime}=\omega / k$ from (10).

Equations (13) - (17) describe the motion of a particle inside the resonant zone. Now let us discuss scattering on resonance and capture into resonance for the problems under consideration in more details.

\subsection{Scattering on resonance.}

The value of $P_{\varphi}$ changes in the process of motion near and inside the resonant zone. The cumulative change in $P_{\varphi}$ during a single passage can be considered as a jump associated with a resonance crossing. In the present paper we calculate this jump using the approach from (Neishtadt, 1999) (in different cases such jump was calculated in (Chirikov, 1959, Kevorkian, 1974)).

In the resonant zone, where $|\dot{\varphi}|<\operatorname{const} \sqrt{\kappa \delta}$, the variables $\theta=k \varphi$ and $\bar{P}_{\varphi} / \sqrt{\kappa \delta}$ change with velocity of order $k \sqrt{\kappa \delta}$ while the variables $\kappa \bar{x}$ and $\bar{P}_{x}$ change with velocity of order $\kappa$ (we assume that $\delta k$ is of order 1). Therefore, in (13) the variables $k \varphi, \bar{P}_{\varphi} / \sqrt{\kappa \delta}$ are fast and the variables $\kappa \bar{x}, \bar{P}_{x}$ are slow. For frozen values of slow variables, dynamics of fast variables is governed by 1D Hamiltonian system with the Hamiltonian $h=h_{\varphi}$ (see (17)):

$$
\dot{\varphi}=g \bar{P}_{\varphi}, \quad \frac{d}{d t} \bar{P}_{\varphi}=-\kappa \frac{\partial h_{\varphi}}{\partial \varphi}=-\kappa b+\kappa k h_{z}^{\prime} \delta \cos (k \varphi) .
$$

Systems of such form, similar to a pendulum with torque, always appear in analysis of resonance crossings (Arnold et al., 1988). A phase portrait of the Hamiltonian $h_{\varphi}$ in the $\left(\varphi, \bar{P}_{\varphi}\right)$ plane can be of one of two types: 

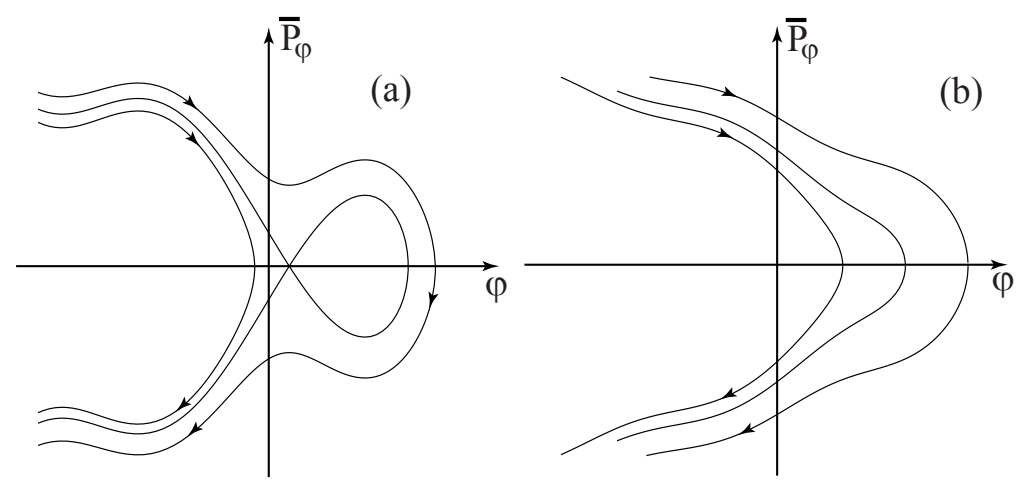

Figure 5: The schematic phase portrait on the $\left(\varphi, \bar{P}_{\varphi}\right)$ plane: (a) $\left|k \delta h_{z}^{\prime}\right| \geq|b|$, (b) $\left|k \delta h_{z}^{\prime}\right|<|b|$. The resonance surface corresponds to $\bar{P}_{\varphi}=0$.

with or without the oscillatory domain, that are shown qualitatively in Figs. 5a and 5b, respectively. Both phase portraits are plotted for $b>0$ and $g>0$. For $b<0$ and $g<0$ we have the opposite direction of motion (in other words, particles move from $\bar{P}_{\varphi}<0$ to $\bar{P}_{\varphi}>0$ ), while for $b>0$ and $g<0$ or $b<0$ and $g>0$ particles come from and leave to the right (in other words, the portrait is mirrored with respect to a vertical axis). In both cases the resonance corresponds to the horizontal axis, $\bar{P}_{\varphi}=0$.

Consider a trajectory of Hamiltonian system (5), that crosses the resonant surface at some time moment $t_{*}$. Denote by $\kappa x_{*}, P_{x, *}, \varphi_{*}$ values of the corresponding variables at $t_{*}$ and let $\theta_{*}=k \varphi_{*}$. Suppose $\theta_{*} \bmod 2 \pi$ is at the distance of order 1 from the root of $\cos \theta=b /\left(\delta k h_{z}^{\prime}\right)$, that corresponds to unstable equilibrium of system (18) with $\kappa \bar{x}=\kappa x_{*}, \bar{P}_{x}=P_{x, *}$ if such an equilibrium exists. Along this trajectory a phase point passes through the resonant zone fast enough, in time of order $1 / \sqrt{\kappa \delta}$. While the phase point moves inside the resonant zone changes of the slow variables are small, of order $\sqrt{\kappa \delta}$. Therefore, for approximate description of dynamics inside the resonant zone one can use equations (18) with $\kappa \bar{x}=\kappa x_{*}, \bar{P}_{x}=P_{x, *}$.

Let $t_{1}$ and $t_{2}$ be two time moments, $t_{1}<t_{*}<t_{2}$ such that $\left|t_{1,2}-t_{*}\right| \sim 1 / \kappa$ and $t_{*}$ is the only moment of crossing of resonance on time interval $\left(t_{1}, t_{2}\right)$. Then the jump of $P_{\varphi}$ on the resonance is

$$
\Delta P_{\varphi}=-\int_{t_{1}}^{t_{2}} \frac{\partial h}{\partial \varphi} d t
$$

where $h$ is given by (5) and values $\kappa x, P_{x}, \varphi, P_{\varphi}$ in the argument of $\partial h / \partial \varphi$ are taken along the exact trajectory; so the integrand is a well defined function of time. In the main approximation, in the argument of $\partial h / \partial \varphi$ one can substitute $\kappa x=\kappa x_{*}, P_{x}=P_{x, *}$ and replace the values of $\varphi$ and $P_{\varphi}$ with the values calculated along a trajectory of (18) with $\kappa \bar{x}=\kappa x_{*}, \bar{P}_{x}=P_{x, *}$ that crosses the resonance at the moment $t_{*}$ having $\varphi=\varphi_{*}$. The integration interval in (19) can be divided into three parts. In the immediate vicinity of the resonance, 
where $\left|t-t_{*}\right|<$ const $1 / \sqrt{\kappa \delta}$, the above approximation works (with an accuracy $O(\kappa \delta)$ ) because dynamics in the resonant zone is described in the main approximation by (18). Relatively far from the resonance, where $\left|t-t_{*}\right| \sim 1 / \kappa$, the approximation works because the integral over this part is small, of order $O(\kappa \delta)$ for both original and modified integral. And in the intermediate part, where const $1 / \sqrt{\kappa \delta}<\left|t-t_{*}\right|<$ const $1 / \kappa$, the approximation works due to a combination of two previous reasons: the larger is $\left|t-t_{*}\right|$ on some part of the integration interval in (19) the worse is approximation of the integrand but the smaller is the value of integral over this part itself. One can show that total accuracy of this approximation is $O(\kappa \delta)$ (see (Neishtadt, 1999)). Replacing in the modified integral the limits $t_{2,1}$ with $\pm \infty$ does not change accuracy. Thus, with such accuracy we get

$$
\Delta P_{\varphi}=-\int_{-\infty}^{\infty} \frac{\partial h}{\partial \varphi} d t=2 \kappa k \delta h_{z}^{\prime} \int_{-s \infty}^{\varphi_{*}} \frac{1}{\dot{\varphi}} \cos (k \varphi) d \varphi,
$$

where the integrand is calculated on the trajectory of (18) as it was discussed above and $s=\operatorname{sign}(b g)$. Substituting $\dot{\varphi}=g \bar{P}_{\varphi}$ (see (18)) into the above equation we get

$$
\Delta P_{\varphi}=2 \kappa k \delta h_{z}^{\prime} \int_{-s \infty}^{\varphi_{*}} \frac{1}{g} \frac{\cos (k \varphi)}{\bar{P}_{\varphi}} d \varphi .
$$

Expressing $\bar{P}_{\varphi}$ in terms of $h_{\varphi}$ and $\varphi$ from (17) we obtain

$$
\Delta P_{\varphi}=2 s \delta k \sqrt{\frac{\kappa}{k}} \frac{h_{z}^{\prime}}{\sqrt{|b g|}} \int_{-s \infty}^{\theta_{*}} \frac{\cos \theta d \theta}{\sqrt{2\left|\frac{h_{\varphi} k}{b}+\delta \frac{h_{z}^{\prime} k}{b} \sin \theta-\theta\right|}}
$$

Substituting $h_{\varphi}$ in terms of $\theta_{*}$ we finally arrive at asymptotic expression:

$$
\Delta P_{\varphi}=2 s \delta k \sqrt{\frac{\kappa}{k}} \frac{h_{z}^{\prime}}{\sqrt{|b g|}} \int_{-s \infty}^{\theta_{*}} \frac{\cos \theta d \theta}{\sqrt{2\left|2 \pi \xi+\delta \frac{h_{z}^{\prime} k}{b} \sin \theta-\theta\right|}},
$$

where the quantity $\xi$, that characterizes a scattering, is given by

$$
2 \pi \xi=\theta_{*}-\delta \frac{h_{z}^{\prime} k}{b} \sin \theta_{*} .
$$

The quantity $\xi \bmod 1$, is a function of the initial data. A small change of order $\kappa$ in the initial conditions produces in general a large change of order 1 in $\xi$. Hence for small $\kappa$ it is the best to treat $\xi$ as a random variable. This approach was suggested in (Lifshitz, et al, 1962) for problems with separatrix crossings. Following (Arnol'd, 1963), see also (Neishtadt, 1999; Itin, 2000), let us determine the distribution of this random variable. Consider a point $M$ far from the resonance such that trajectory passing through $M$ intersects the resonance. Let $V_{d}$ be a sphere of radius $d$ centered at $M$. Assume that each point of this sphere is an initial point of a trajectory. 
Denote by $V_{d, \kappa}^{(a, b)}$ the part of the sphere $V_{d}$ for whose points the quantity $\xi$ lies in the interval $(a, b) \in(0,1)$. Define the probability for $\xi$ to fall into the interval $(a, b)$ as

$$
P\{\xi \in(0,1)\}=\lim _{d \rightarrow 0} \lim _{\kappa \rightarrow 0} \frac{\operatorname{vol} V_{d, \kappa}^{(a, b)}}{\operatorname{vol} V_{d}}
$$

where the symbol vol represents the standard volume in $\mathbb{R}^{4}$. It can be shown that $P\{\xi \in(0,1)\}=(b-a)$ (see (Neishtadt, 1999)). Therefore, $\xi$ can be treated as a random variable uniformly distributed on $(0,1)$. This property was checked numerically in (Itin, 2000)).

Statistical properties of the scatterings depend on the shape of the phase portrait in the $\left(\varphi, \bar{P}_{\varphi}\right)$ plane. The relation between the values of $b$ and $k \delta h_{z}^{\prime}$ specify which of two types is realized. If

$$
\left|k \delta h_{z}^{\prime}\right| \geq|b|
$$

the phase portrait looks like the one shown in Fig. 5 a and, in a generic case, scatterings on resonance cause $P_{\varphi}$ diffuse and drift. The rate of drift is determined by the ensemble average of $\Delta P_{\varphi}$ :

$$
\left\langle\Delta P_{\varphi}\right\rangle=\int_{0}^{1} \Delta P_{\varphi}(\xi) d \xi
$$

It was shown in (Neishtadt, 1999) that

$$
\left\langle\Delta P_{\varphi}\right\rangle=-\frac{1}{2 \pi} \operatorname{sign}(b) k S_{R},
$$

where $S_{R}$ is the area under the separatrix loop in Fig. 5a:

$$
S_{R}=2 \frac{1}{k} \sqrt{\frac{\kappa}{k}} \int_{\theta_{\min }}^{\theta_{\max }}\left(2 \frac{b}{g}\left(2 \pi \xi_{c}+\frac{\delta h_{z}^{\prime} k}{b} \sin \theta-\theta\right)\right)^{1 / 2} d \theta .
$$

The integral in (23) is taken over the interval where the radicand expression is positive. The quantity $\xi_{c}$ is given by

$$
2 \pi \xi_{c}=-\frac{\delta h_{z}^{\prime} k}{b} \sin \theta_{c}+\theta_{c},
$$

where $\theta_{c}$ is the value of $\theta$ at the hyperbolic fixed point in Fig. 5a. One can see that $\theta_{c}=\theta_{\min }$ for $b g<0$ and $\theta_{c}=\theta_{\max }$ for $b g>0$. Order of magnitude estimates yield

$$
\left\langle\Delta P_{\varphi}\right\rangle \sim \sqrt{\kappa \delta} .
$$

Similarly, rate of diffusion depends on the mean square value of $\Delta P_{\varphi}$ :

$$
D=\int_{0}^{1}\left(\Delta P_{\varphi}(\xi)-\left\langle\Delta P_{\varphi}\right\rangle\right)^{2} d \xi \sim \kappa \delta .
$$


The long-term rate of drift depends on the quantity $\left\langle\Delta P_{\varphi}\right\rangle_{\Sigma}$, that is equal to the sum of all the $\left\langle\Delta P_{\varphi}\right\rangle$ during one period of $x$-motion. It is clear that two successive crossings of a connected subset of $\mathrm{R}$ by a trajectory of $x$ motion occur in the opposite directions. Hence, the corresponding values of $b$ have the opposite signs. Therefore, it follows from (22) that, if $\left\langle\Delta P_{\varphi}\right\rangle \neq 0$, the corresponding values of $\left\langle\Delta P_{\varphi}\right\rangle$ have the opposite signs.

If (21) does not hold, the phase portrait looks like the one shown in Fig. 5b. In this case, the mean change of $P_{\varphi}$ is zero: as there is no separatrix, $S_{R}=0$. The evolution of $P_{\varphi}$ is purely diffusive.

Turn to the particular cases considered above.

1. $k_{y}=0$.

In this case

$$
b=h_{z}^{\prime}, \quad g=1,
$$

and condition (21) is reduced to

$$
k \delta \geq 1
$$

If the above inequality holds we have

$$
\begin{aligned}
\left\langle\Delta P_{\varphi}\right\rangle & =-\frac{1}{2 \pi}\left(\operatorname{sign}\left(h_{z}^{\prime}\right)\right) k S_{R} \\
S_{R} & =2 \frac{1}{k} \sqrt{\frac{\kappa}{k}} \sqrt{\left|h_{z}^{\prime}\right|} \int_{\theta_{\min }}^{\theta_{\max }}\left(2\left|2 \pi \xi_{c}+k \delta \sin \theta-\theta\right|\right)^{1 / 2} d \theta .
\end{aligned}
$$

Therefore, $\left\langle\Delta P_{\varphi}\right\rangle>0$ if $h_{z}^{\prime}<0$ and $\left\langle\Delta P_{\varphi}\right\rangle<0$ if $h_{z}^{\prime}>0$. As two consecutive scatterings occur at the same value of $P_{x}>0$ (recall, that in this particular case the projection of $R$ on the $\left(\kappa x, P_{x}\right)$ plane is a straight line parallel to the $\kappa x$-axis), it follows from Fig. 3 and Fig. 4 , that for the particles with large energy the average change of $P_{\varphi}$ on one turn of $x$-motion is positive: $\left\langle\Delta P_{\varphi}\right\rangle_{\Sigma}>0$. Consequently, the energy of particle increases.

2. $k_{x}=0$.

In this case

$$
b=P_{x}, \quad g=h_{z}^{\prime \prime}
$$

Note, that in this case the phase portrait of $x$-motion (Fig. 4) is symmetric with respect to the abscissas axis. Hence, the values of $\left\langle\Delta P_{\varphi}\right\rangle$ at two intersections of a trajectory of $x$-motion with $\mathrm{R}$ compensate each other, $\left\langle\Delta P_{\varphi}\right\rangle_{\Sigma}=0$, and the statistics of the changes of $P_{\varphi}$ is diffusive, regardless of the validity of (21). 


\subsection{Capture into resonance.}

The other phenomenon that affects the dynamics of a particle at a resonance crossing is capture into resonance. Due to nonuniformity of the magnetic field, the captured dynamics is quite rich comparing with the previous publications (see e.g. Itin et al., 2000)). While the conditions of capture and release and the conservation laws that govern the captured motion remain the same, properties of the input-output function (the value of $P_{\varphi}$ after release, $P_{\varphi, f i n}$, as a function of initial value of $P_{\varphi}, P_{\varphi, \text { in }}$, other phase variables and parameters of the wave) are more complex. In (Itin et al., 2000), $P_{\varphi, f i n}$ was either (approximately) equal to $P_{\varphi, \text { in }}$ if the resonant curve was an ellipse, or went to infinity if the resonant curve was a parabola or a hyperbola. In our case, the value of $P_{\varphi, \text { fin }}$ depends strongly, among other parameters, on the direction of the wave vector, $k$, with the possibilities of $P_{\varphi, \text { fin }}$ returning to $P_{\varphi, \text { in }}$, going to the infinity or anything in between.

Capture is possible only if the phase portrait in the $\left(\varphi, \bar{P}_{\varphi}\right)$ plane looks like the one shown in Fig. 5a, in other words, if $(21)$ is satisfied and the phase space $\left(\varphi, \bar{P}_{\varphi}\right)$ has a well-defined separatrix. In this case, the expectation of the change of $P_{\varphi}$ (the probability of a phenomenon times the size of a change of $P_{\varphi}$ ) is the same for scattering and capture.

Capture into resonance can be described as follows. The area under the separatrix loop, $S_{R}$, in the $\left(\varphi, \bar{P}_{\varphi}\right)$ plane changes as a particle moves along a phase trajectory. Suppose the area under the separatrix loop increases when the particle approaches the resonance. Then if the particle comes very close to the hyperbolic fixed point it may cross the separatrix and, as a result, be caught in the oscillatory domain within the separatrix loop. In this case, the particle starts moving on the resonant surface and it's motion is governed by the conservation law

$$
P_{\varphi}=P_{\varphi, r e s}\left(\kappa x, P_{x}\right)
$$

or, in other words, by the Hamiltonian $h_{x}$ (see (14)). The area under a phase trajectory in the $\left(\varphi, \bar{P}_{\varphi}\right)$ plane remains constant. As a result, the captured motion is integrable. While the particle moves, the area under the separatrix loop, that is a function of slow variables, changes and, if it returns to the initial value, the particle is released from the resonance. If the area under the separatrix loop keeps increasing, the captured particle accelerates along $y$-axis until it exits the system.

As it was discussed in Sect. 4, capture can be considered as a probabilistic phenomenon: initial conditions for particles that are or are not captured are mixed. Consider a point $M$ far from the resonance such that the trajectory passing through $M$ intersects the resonance. Let $V_{d}$ be a sphere of radius $d$ centered at $M$ and $V_{d, \kappa}^{c}$ be the part of $V_{d}$ formed by initial conditions of trajectories with a capture into the resonance (see (Itin, 2000)). 
We define the probability of capture at $M$ as

$$
P(M)=\sqrt{\kappa \delta} \lim _{d \rightarrow 0} \lim _{\kappa \rightarrow 0} \frac{\operatorname{vol} V_{\delta, \kappa}^{(a, b)} / \sqrt{\kappa \delta}}{\operatorname{vol} V_{\delta}} .
$$

Following (Neishtadt, 1999), we have (see (23)):

$$
P(M)=k \frac{\left\{S_{R}, h_{r e s}\right\}_{*}}{2 \pi|b|_{*}} \sim \sqrt{\kappa \delta}, \quad \text { if }\left\{S_{R}, h_{r e s}\right\}_{*}>0,
$$

where $h_{\text {res }}$ was defined in $(11),\{$,$\} denotes Poisson bracket and the subscript ' *$ ' indicates that the corresponding quantity must be evaluated at the resonance. For $\left\{S_{R}, h_{r e s}\right\}_{*}<0, P(M)=0$.

Turn to two particular cases discussed above.

1. $k_{y}=0$.

In this case, the capture is possible if $(\omega / k) h_{z}^{\prime}(\kappa x) h_{z}^{\prime \prime}(\kappa x)>0$, which is the condition for $\dot{S}_{R}>0$. For a wave propagating in the direction opposite to $x$-axis (from the earth), when $\omega / k<0$, this condition is satisfied on two intervals: $\kappa x<\zeta_{m}$ and $\kappa x>\zeta_{s}$ (see Fig. 3). Particles which were captured on the interval $\kappa x<\zeta_{m}$ accelerate up to the boundary of our 2D system in $y$-direction. Particles which are captured on the interval $\kappa x>\zeta_{s}$ are transported by the wave into the region $\zeta_{m}<\kappa x<\zeta_{s}$ where they are released from the resonance. For a wave propagating along $x$-direction (towards the Earth in the magnetotail) $(\omega / k>0)$, the capture is possible on the interval $\zeta_{m}<\kappa x<\zeta_{s}$. The wave transports captured particles into the region $\kappa x>\zeta_{s}$ where the particles are released from the resonance.

Consider the case $\omega / k<0$ in more detail. A typical dynamics of a particle is shown in Fig. 6. In Fig. 6a, a particle moves from the rightmost point along the averaged trajectory and is captured into the resonance $\left(h_{z}^{\prime}\right.$ is increasing, hence the area under the separatrix loop is increasing: see (24) and Fig. 3). The captured particle is transported to the region where $h_{z}^{\prime}$ is decreasing and the particle is released from the resonance and starts moving along another trajectory of the averaged system. The next capture is shown in Fig. 6b. In this case, however, the area under the separatrix loop increases unboundedly (within the limits of the validity of our model) and the particle is transported to the edge of the system. Figure $6 \mathrm{c}$ presents the phase portrait on the $\left(\varphi, P_{\varphi}\right)$ plane for the last part of the trajectory. $P_{\varphi}$ is nearly constant when a particle moves far from the resonance, undergoes a jump when the trajectory is scattered crossing $R$ and goes towards $-\infty$ during the captured motion. It follows from $(2)$, that the sum of the energy of a particle and $(-\omega / k) P_{\varphi}$ is constant. Therefore, if $P_{\varphi}$ is decreasing towards $-\infty$ (see Fig. 6c), energy increases up to $H_{\max }=E L_{y}$, where $L_{y}$ is the transverse dimension of the system, when a captured particle leaves the physical boundaries of the system. 

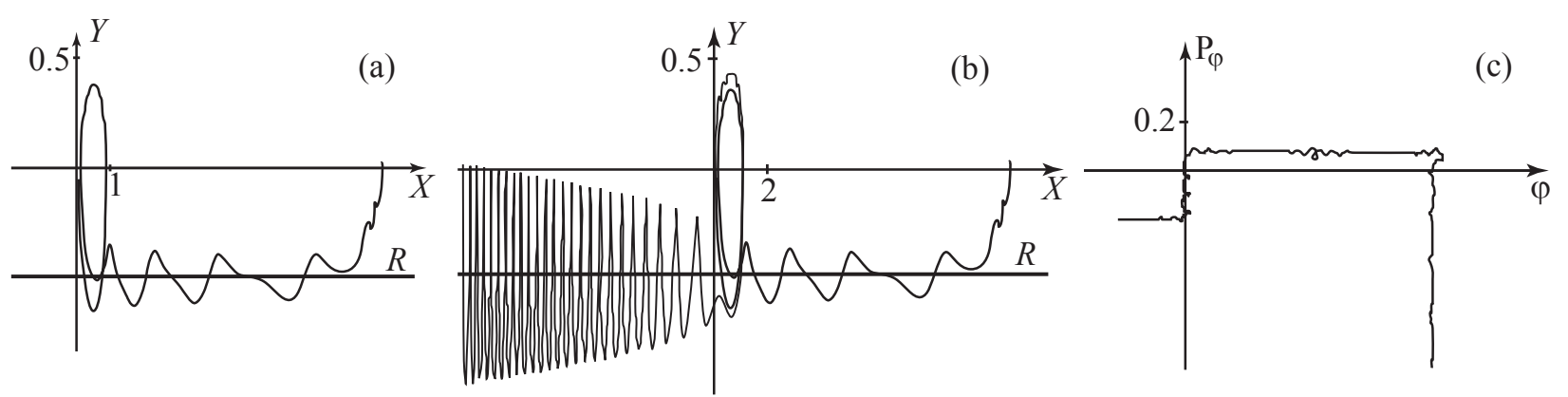

Figure 6: The projection of a long-time trajectory of a particle on the $(X, Y)$ plane (see $(7))$ in the case $k_{y}=0$, electromagnetic wave. (a) The capture into resonance and the subsequent release from resonance, (b) the next capture and the subsequent transport to the infinity, (c) the projection of motion on the $\left(\varphi, P_{\varphi}\right)$ plane.

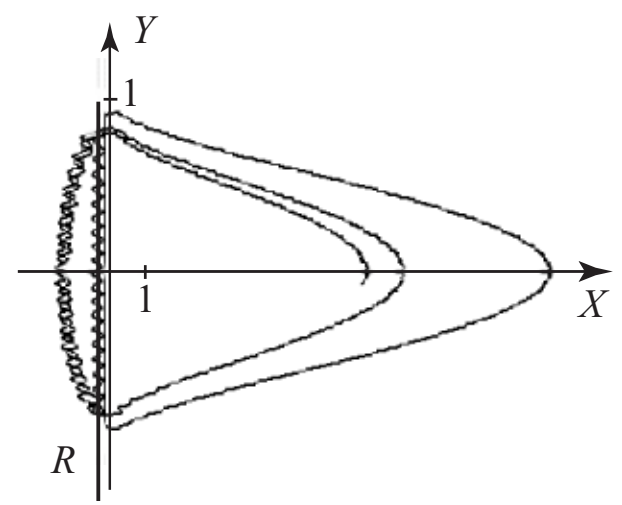

Figure 7: The projection of a long-time trajectory of a particle on the $(X, Y)$ plane in the case $k_{x}=0$ for electromagnetic wave.

2. $k_{x}=0$.

The section of $R$ by a plane $P_{\varphi}=$ const is either empty or has the form of one or two straight lines that are parallel to the $P_{x}$-axis. It follows from the symmetry of the phase portrait of $x$-motion with respect to the axis of abscissas that a capture into the resonance and the consequent release from the resonance occur at the same value of $P_{x}$. The capture happens in the upper half-plane if $\omega / k<0$ and in the lower half-plane if $\omega / k>0$. Figure 7 presents the projection of the phase curve onto the $(X, Y)$ plane. It illustrates that the capture into the resonance is a probabilistic process: the capture occurs only once in three consecutive crossings of $R$. The other two crossings result in the scattering on the resonance.

Finally, Fig. 8 presents the projection of one phase curve onto the $(X, Y)$ plane in the general case $k_{y} \neq 0$, $k_{x} \neq 0$. The particle is captured into the resonance and is transported to the edge of the system. 


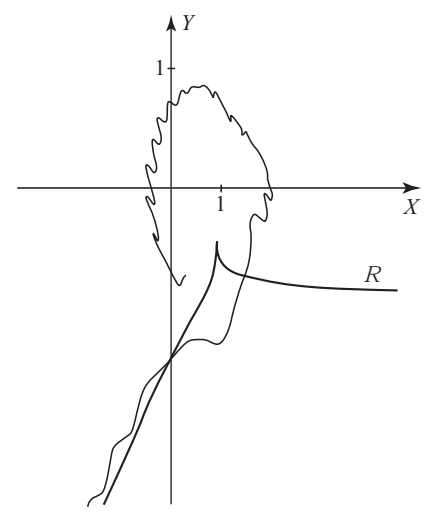

Figure 8: The dynamics of a particle in a generic case of the electromagnetic wave $\left(k_{y} \neq 0, k_{x} \neq 0\right)$ : the capture into resonance and the transport to the infinity.

\section{Dynamics of particles in the presence of an electrostatic wave.}

Now let us derive an analogue of Hamiltonian (5) for an electrostatic wave. Recall that a wave is called electrostatic if the vector of the electric field is parallel to the wave vector. In this case, there is no perturbation of the magnetic field. Such a wave can be described by a scalar potential

$$
\Phi=\frac{E}{\bar{k}} \sin (\overline{\mathbf{k}} \mathbf{x}-\bar{\omega} t)
$$

The induced electric field is given by $\mathbf{E}=\operatorname{grad} \Phi$ and the Hamiltonian takes the form

$$
H=\frac{1}{2 m}\left\{P_{x}^{2}+\left[P_{y}-\frac{e}{c}\left(B_{n} x-B_{0} \frac{z^{2}}{2 L}\right)\right]^{2}+P_{z}^{2}\right\}+e \Phi .
$$

Applying normalizing conditions (1), introducing the phase $\varphi$ and averaging over $z$-motion we arrive at

$$
\begin{aligned}
h & =\frac{1}{2}\left(P_{x}+\frac{k_{x}}{k} P_{\varphi}\right)^{2}-\frac{\omega}{k} P_{\varphi}+h_{z}\left(-\frac{k_{y}}{k} P_{\varphi}+\kappa x\right)+\kappa \frac{\varepsilon}{k} \sin (k \varphi) . \\
& =h_{a v}\left(\kappa x, P_{x}, P_{\varphi}\right)+\kappa \frac{\varepsilon}{k} \sin (k \varphi) .
\end{aligned}
$$

One can see that (5) and (26) are very similar. In particular, the function $h_{a v}$ is the same for both Hamiltonians. Hamiltonian (26) differs from Hamiltonian (5) by the substitution of $\kappa \frac{\varepsilon}{k} \sin (k \varphi)$ instead of $-\kappa \frac{\varepsilon}{\omega} h_{z}^{\prime} \sin (k \varphi)$. Therefore, $h_{a v}$ does not change and the equation for the resonant surface and the expression for $h_{x}$, (Eq.(14)), are the same for electrostatic wave as for electromagnetic wave. The equation for $h_{\varphi}$ takes the form

$$
\kappa h_{\varphi}=\kappa b \varphi+\frac{1}{2} g \bar{P}_{\varphi}^{2}+\kappa \frac{\varepsilon}{k} \sin (k \varphi),
$$


where $b$ and $g$ are given by (16).

\subsection{Scattering on resonance.}

The expression analogous to (20) for an electrostatic wave is

$$
\Delta P_{\varphi}=-2 \varepsilon s \sqrt{\frac{\kappa}{k}} \frac{1}{\sqrt{|b g|}} \int_{-s \infty}^{\theta_{*}} \frac{\cos \theta d \theta}{\sqrt{2\left|2 \pi \xi-\frac{\varepsilon}{b} \sin \theta-\theta\right|}},
$$

where $\xi$ is given by

$$
2 \pi \xi=\frac{\varepsilon}{b} \sin \theta_{*}+\theta_{*} .
$$

The error term in (28) is of order $\kappa / k$, provided $\varepsilon$ is of order 1 and $\theta_{*} \bmod 2 \pi$ is at the distance of order 1 from the root of equation $\sin \theta=b / \varepsilon$, that correspond to unstable equilibrium position of system (27). The condition analogous to (21) takes the form

$$
\varepsilon \geq|b| .
$$

The ensamble average of the jumps of $P_{\varphi}$ is:

$$
\begin{aligned}
\left\langle\Delta P_{\varphi}\right\rangle & =-\frac{1}{2 \pi} \operatorname{sign}(b) k S \\
S_{R} & =2 \frac{1}{k} \sqrt{\frac{\kappa}{k}} \int_{\theta_{\min }}^{\theta_{\max }}\left(2 \frac{b}{g}\left(2 \pi \xi_{c}-\frac{\varepsilon}{b} \sin \theta-\theta\right)\right)^{1 / 2} d \theta .
\end{aligned}
$$

Turn to the particular cases considered above.

1. $k_{y}=0$.

In this case the ensamble average of the jumps of $P_{\varphi}$ is

$$
\begin{aligned}
\left\langle\Delta P_{\varphi}\right\rangle & =-\frac{1}{2 \pi} \operatorname{sign}\left(h_{z}^{\prime}\right) k S, \\
S_{R} & =2 \frac{1}{k} \sqrt{\frac{\kappa}{k}} \sqrt{\left|h_{z}^{\prime}\right|} \int_{\theta_{\min }}^{\theta_{\max }}\left(2\left|2 \pi \xi_{c}-\frac{\varepsilon}{h_{z}^{\prime}} \sin \theta-\theta\right|\right)^{1 / 2} d \theta .
\end{aligned}
$$

Unlike the case of an electromagnetic wave, the area under the separatrix loop decreases as $\left|h_{z}^{\prime}\right|$ increases. We also have that $\left\langle\Delta P_{\varphi}\right\rangle>0$ if $h_{z}^{\prime}<0$ and $\left\langle\Delta P_{\varphi}\right\rangle<0$ if $h_{z}^{\prime}>0$. It follows from Fig. 3 , that for the same value of $h_{z}$ the value of $\left|h_{z}^{\prime}\right|$ is larger if $h_{z}^{\prime}<0$. Therefore, the average change of $P_{\varphi}$ on one period of $x$-motion is negative: $\left\langle\Delta P_{\varphi}\right\rangle_{\Sigma}<0$. Hence, the energy of the particles that cross the resonance decreases.

2. $k_{x}=0$. 


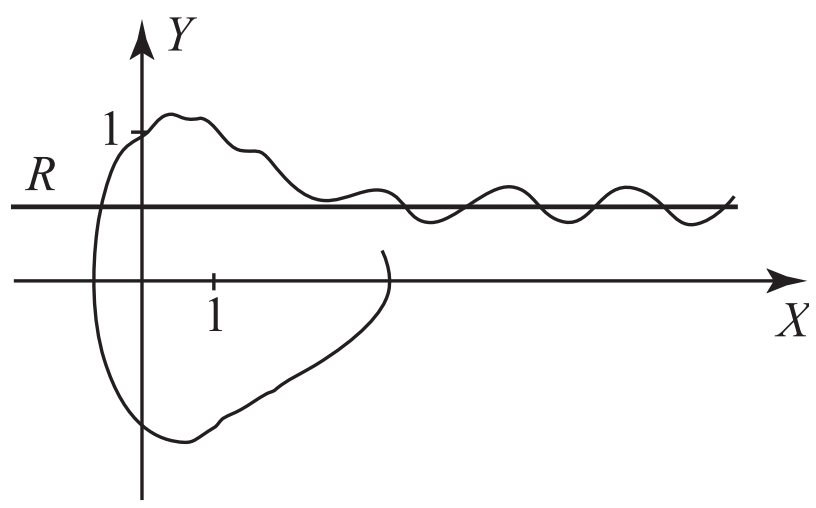

Figure 9: The scattering on resonance and the subsequent capture into resonance for an electrostatic wave in the case $k_{y}=0$.

In this case there is no difference between the interaction particles with electrostatic and electromagnetic waves.

\subsection{Capture into resonance.}

Let us turn to capture into the resonance and focus on the $k_{y}=0$ case. Recall, that the intersection of the resonant surface and the $P_{\varphi}=$ const plane is a straight line, parallel to the $\kappa x$-axis. Contrary to the case of electromagnetic wave (Section 5.2), capture into resonance is possible if $\left(\omega / k_{x}\right) h_{z}^{\prime}(\kappa x) h_{z}^{\prime \prime}(\kappa x)<0$. Particles are captured into the resonance with waves moving from the Earth $\left(\omega / k_{x}<0\right)$ within the interval $\zeta_{m}<\kappa x<\zeta_{s}$. The captured particles are transported into the region $\kappa x<\zeta_{m}$ and are released from the resonance there. The dynamics in this case is shown in Fig. 9. Particles can be captured into a wave that is moving towards the Earth $\left(\omega / k_{x}>0\right)$ in the regions $\kappa x>\zeta_{s}$ and $\kappa x<\zeta_{m}$. The particles that were captured in the region $\zeta_{-}<\kappa x<\zeta_{m}$ are transported into the region $\zeta_{m}<\kappa x<\zeta_{s}$ and are released from the resonance there. Here $\zeta_{-}$is given by the conditions $\zeta_{-}<\zeta_{s}$, and $\left|h_{z}^{\prime}\left(\zeta_{-}\right)\right|=h_{z}^{\prime}\left(\zeta_{s}\right)$. Other captured particles stay in the resonance and are transported to the edge of the system.

\section{The long-time behaviour of the particles.}

The earth magnetotail is an open system. Charged particles from the solar wind enter in the far region (see Fig. 1a) and then accelerate and drift towards the earth. Characteristic life time of particles in the magnetotail, $T_{L}$, is defined by the magnitude of the constant solar wind-induced electric field and non-uniformity of magnetic field (see Sect. 2 and e.g. Vainshtein et al., 1995). In the present section we discuss the properties of particles' 
dynamics on the time scales of order of $T_{L}$, that spans many resonance and separatrix crossings. For a typical particle, $T_{L}$ is much longer then the period of the $x$-motion, that is the slowest motion in the system.

In the region where there are neither resonance nor separatrix crossings the phase space is filled by invariant tori up to a residue of exponentially small measure and the value of both adiabatic invariants, $I_{z}$ and $P_{\varphi}$, are conserved eternally: on infinite time interval it has only oscillations of order $\kappa$ (the Arnold theorem about perpetual adiabatic invariance, see, e.g. (Arnold et al., 1988)).

The accumulation of the jumps of adiabatic invariants could produce chaotic dynamics. In the case of separatrix crossings for the problem under consideration it was illustrated in (Büchner and Zelenyi, 1989; Zelenyi et al., 1990). Long-term evolution of adiabatic invariants depends on whether consecutive crossings are statistically dependent or independent. Statistical independence follows from the divergence of phases along trajectories. For separatrix crossings consecutive crossings for some initial conditions are statistically dependent (Cary and Skodje, 1989) and islands of stability, albeit being of a small measure, do exist (Elskens and Escande, 1991; Neishtadt et al, 1997) inside large chaotic see. Consecutive resonance crossings should be treated as independent as shows the following reasoning from (Neishtadt, 1999).

Let two successive crossings of the resonant surface occur at $\varphi_{1}$ and $\varphi_{2}$. A small variation $\Delta \varphi_{1}$ of $\varphi_{1}$ produces the variation of the jump value of $P_{\varphi}$ by a quantity of order $\sqrt{\kappa \delta} \Delta \varphi_{1}$. As a result, the phase $\varphi_{2}$ changes by the quantity $\left.\Delta \varphi_{2} \sim(1 / \kappa \delta) \sqrt{\kappa \delta}\right) \Delta \varphi_{1}=(1 / \sqrt{\kappa \delta}) \Delta \varphi_{1} \gg \Delta \varphi_{1}$. In other words, a small change in the resonance phase before one crossing generally results in a big change in the value of the resonance phase of the next crossing. Therefore, jumps of adiabatic invariant at two successive crossings can be considered as independent.

With consecutive jumps being independent, evolution of the adiabatic invariant due to multiple passages through the resonance can be treated a random walk with a step of order of $\sqrt{\kappa \delta}$ with or without a preferred direction depending of the validity of (21). In the absence of the drift (i.e. no preferred direction), diffusion of adiabatic invariants is observed in (nearly all) numeric simulations. Diffusion time can be estimated as a product of a characteristic mean square value of a jump and a characteristic slow period. Thus estimated, diffusion time was shown to be in a good agreement with numerical simulations (see (Bruhwiler and Cary, 1989) and (Itin, 2000) for separatrix and resonance crossings, respectively).

The assumption of statistical independence is crucial for chaotization: when phases on the successive crossings are related a regular motion can emerge. Such a phenomenon may appear if $T_{x}$ is (approximately) independent of $P_{\varphi}$. In this case $\partial T_{x} / \partial P_{\varphi}$ vanish and consecutive phases become correlated. In particular, consecutive 
jumps may cancel each other - a phenomenon called beamlets (see e.g. Chen, 1992, Ashour-Abdalla, 1993), that is well known in magnetospheric applications. Beamlets occur on short intervals in history of particle convection from the distant tail to the earth. For nearby trajectories net effect (averaged for all the crossings during one period of $x$-motion), although not exactly vanishing, is much smaller than in a regular case. As a mixing parameter, $\eta$ (see below), is proportional to the square of the average net change, it nearly vanishes in the vicinity of beamlet trajectories and particles behave as if they are in an adiabatic regime.

\subsection{Loss of adiabatic invariancy and mixing over a finite time.}

In the chaotic region resonance and separatrix crossings lead to loss of adiabatic invariancy, which, in turn, results in mixing. Had the particles stay in the magnetotail forever, the result would be complete mixing and washing out of all the structures in the distribution functions of the chaotic region that were present when particles entered the magnetotail.

To define a measure of importance of resonance and separatrix crossings we introduce the characteristic time of mixing due to either process, $T_{r}$ and $T_{j}$, respectively, as the time over which the diffusion spreads the value of the adiabatic invariant $\left(P_{\varphi}\right.$ or $I_{z}$, respectively) by a quantity of order 1 (see below). The combined extend of mixing in the system depends on the relation between the $T_{r}$ and $T_{j}$ on one hand and $T_{L}$ on the other. If both $T_{r}$ and $T_{j}$ are larger then $T_{L}$, then mixing is small everywhere in the phase space and distribution functions are transported towards the earth relatively intact. Contrarily, if at least one of $T_{r}$ or $T_{j}$ is less then $T_{L}$, then mixing is important and distribution functions are homogenized. The relative importance of resonant phenomena and the jumps of the adiabatic invariant depends on the comparison between $T_{r}$ and $T_{j}$. To be definite, in what follows we consider the case of the electromagnetic wave.

As the phenomena related to both resonant and separatrix crossings are localized near $R$ and UC, respectively, characteristic time of mixing due to either process can be written as

$$
T_{r(j)} \sim T_{p e r} \frac{1}{D}
$$

In (29), $T_{p e r}$ is a typical time between consecutive crossings of $R$ or $\mathbf{U C}$ and is of order of the period of the $x$-motion. $D$ is the mean square value of a jump of the adiabatic invariant in a single passage (of order of the square of a characteristic jump).

The value of $T_{p e r}$ is the same for both separatrix and resonance crossings and is of order of $\sim 1 / \kappa$. It was shown in (Zelenyi et al., 1990; Vainshtein et al., 1995), that separatrix crossings lead to the diffusion of the 
adiabatic invariant with a characteristic size of jumps of order $\sim \kappa$. Therefore,

$$
T_{j} \sim \kappa^{-3}
$$

For resonant phenomena $D \sim \kappa \delta$. Hence,

$$
T_{r} \sim \frac{1}{\kappa}(\kappa \delta)^{-1}
$$

and we arrive at

$$
\frac{T_{r}}{T_{j}} \sim \frac{\kappa^{3}}{\delta \kappa^{2}}=\frac{\kappa}{\delta} .
$$

The value of $T_{r} / T_{j}$ defines what phenomenon is more important for the chaotization of the particles. If $T_{r} / T_{j} \ll 1$, the resonant processes dominate. If $T_{r} / T_{j} \gg 1$, the separatrix crossings dominate. Note, that $\kappa$ is a function of the distance from the earth while $\delta$ is a parameter of a wave. Therefore, $T_{r}<T_{j}$ defines a range of parameters of the waves that must be taken into account when describing the long-time dynamics of charged particles in a particular part of the magnetotail.

To describe the extend of the overall mixing we introduce a parameter

$$
\eta=\frac{T_{L}}{\min \left(T_{j}, T_{r}\right)} .
$$

If $\eta \ll 1$, the mixing is negligible. If $\eta \gg 1$, the mixing is strong: particles cross resonance (or separatrix) sufficient number of times for adiabatic invariant cover the whole admissible domain. A similar observation (for separatrix crossings) was made in (Karimabadi et al., 1990).

\subsection{Resonances and acceleration of particles.}

There is an additional major difference between the impacts of resonance phenomena and separatrix crossings. Unlike separatrix crossings, resonance phenomena can on average lead to acceleration of particles. If the average value of $\left\langle\Delta P_{\varphi}\right\rangle$ during a period of $x$-motion, $\left\langle\Delta P_{\varphi}\right\rangle_{\Sigma}$, does not vanish, there is an average drift of energy (see $(2))$ :

$$
\frac{d h}{d t}=\frac{1}{T_{p e r}}\left\langle\Delta P_{\varphi}\right\rangle_{\Sigma} \frac{\omega}{k} .
$$

For $\left\langle\Delta P_{\varphi}\right\rangle_{\Sigma}$ to be non-zero, (21) must be valid. For electromagnetic wave $\left\langle\Delta P_{\varphi}\right\rangle_{\Sigma} \sim \sqrt{\kappa \delta}$ (see Subsect. 5.1). Taking (1) and the estimate for $T_{p e r}$ into account, we arrive at

$$
\frac{d h}{d t} \sim \kappa \sqrt{\kappa \delta} \frac{\omega}{k} .
$$

Note, that although it was omitted in (30), the value of $\left\langle\Delta P_{\varphi}\right\rangle_{\Sigma}$ depends on the direction of the wave vector. In particular, as it was shown in Subsect. 5.1, $\left\langle\Delta P_{\varphi}\right\rangle_{\Sigma}=0$ for $k_{x}=0$ and there is no acceleration. Numerical 
simulations show that for electromagnetic wave $\left\langle\Delta P_{\varphi}\right\rangle_{\Sigma}$ is always non-negative and reaches its maximum and minimum (zero) values for $k_{y}=0$ and $k_{x}=0$, respectively. Therefore, electromagnetic waves accelerate particles.

On the other hand, for electrostatic waves $\left\langle\Delta P_{\varphi}\right\rangle_{\Sigma}$ is always non-positive, vanishing for $k_{x}=0$ and reaching the maximum absolute value for $k_{y}=0$ (see Sect. 6). In this case, instead of (30), we have

$$
\frac{d h}{d t} \sim-\kappa \sqrt{\kappa \varepsilon / k} \frac{\omega}{k}
$$

and the energy of particles decrease.

\section{Discussion and conclusions.}

In the present paper we discuss the influence of a single low-frequency monochromatic electromagnetic or electrostatic wave on the dynamics of charged particles and mixing in the magnetic-field-reversal region of the earth magnetotail. Assuming that the amplitude of a wave is small and the frequency resides between the characteristic frequencies of the motion in and perpendicular to the equatorial plane we solved the problem using perturbation theory.

We show that waves effect the dynamics of the particles mostly by the way of resonant phenomena: scattering on resonance and capture into resonance. Each passage through resonance results in an abrupt change in the adiabatic invariant. We derive expressions for jumps of adiabatic invariants and show that in the case of multiple passage through resonance these phenomena can be treated as random processes. Resonance phenomena, like the jumps of the adiabatic invariant on a separatrix, lead to the chaotization of motion and mixing. We estimate the characteristic times of mixing due to resonance and separatrix phenomena, discuss their relative importance on the dynamics of the particles and introduce a parameter that can serve as a measure of mixing. We calculate the range of the parameters of the problem when the resonant phenomena are the primary mechanisms of the chaotization. We show that besides the chaotization the resonant phenomena can lead to a significant acceleration of particles limited, for some values of the parameters of a wave, only by the scales of the system.

We would like to link the strong ("unbound", in terms of our model) particle acceleration with the discovery of the so-called magnetotail beamlets (Grigorenko, et al., 2002). Beamlets represent short (tens of seconds) spikes of highly accelerated particles usually registered at magnetotail edges. This phenomenon could hardly be explained by standard laminar models of acceleration. Some strong transient effects are obviously involved in their formation. Resonant interactions of particles with electromagnetic or electrostatic waves could provide 
the plausible mechanism of such a bursty particle energization.

Let us discuss the validity of the restrictions we put on the wave. The magnetic field in the magnetotail is quite non-uniform in the $z$-direction. The typical length scale of the non-uniformities is of order $L_{z} \sim 1 R_{E}$, where $R_{E}$ is the radius of the earth. The assumption that waves propagate in the equatorial plane $\left(k_{z}=0\right)$ is motivated by very significant confinement of particles in the vicinity of the current sheet: strong and fast bounce-like motion of particles across the sheet makes the interaction with the waves propagating in the current sheet the most effective. The second assumption, that the wave is monochromatic, given that it propagates in the $(x, y)$ plane, is less restricting. Typical sizes of the magnetotail in the $y$ - and $x$ - directions are relatively large: $L_{y} \sim 30 R_{E}$, and $L_{x} \sim 100 R_{E}$, hence the system is approximately uniform for the perturbations with the wave length $\lambda \ll L_{y}, L_{x}$.

Finally, the results obtained for a single wave can be easily generalized for a case of wave packets propagating in the current sheet, as any given wave appreciably effects the motion of a particle only when a particle is in the resonance with this particular wave. Numerical simulations of interaction between particles and wave packets performed during last few years support this statement. Although wave packets with random phases usually make particle trajectories within magnetic field reversal very chaotic, one could still see the evidence of resonant-like effects manifested as large scale Levy flights (Veltri, et al., 1998; Greco, et al., 2001).

\section{Acknowledgments}

This work was performed with the financial support of Russian Basic Research Foundation Grant No. 00-0100538 and INTAS Grant No. 00-221 (A.N., E.R., and D.V.); and Russian Basic Research Foundation Grant No. 00-15-96631, No. 01-02-16367 and No. 03-01-00158 and INTAS Grant No. 99-0078 (L.Z.). L.Z. is also grateful to the Humboldt foundation for the support.

\section{References}

Arnol'd V.I., Small denominators and problems of stability of motion in classical and celestial mechanics, Russ. Math. Surveys, 18, 85-192, 1963.

Arnold, V.I., Kozlov, V.V. and Neishtadt, A.I., Dynamical Systems III. Encyclopedia of Mathematical Sciences, Springer-Verlag; New York, N.Y., 1988.

Ashour-Abdalla, M., Büchner, J., and Zelenyi, L.M., The quasi-adiabatic ion distributions in the central plasma sheet and its boundary layer, J. Geophys. Res., 96, 1601-1610, 1991. 
Ashour-Abdalla, M., Berchem, J.P., Büchner, J., and Zelenyi, L.M., Shaping of the magnetotail from the mantle - global and local structuring, J. Geophys. Res., 98, 5651-5676, 1993.

Ashour-Abdalla, M., Peroomian, V., Richard, R., and Zelenyi, L.M., The mosaic structure of plasma bulk flows in the Earth's magnetotail, J. Geophys. Res., 100, 19191-19209, 1995.

Ashour-Abdalla, M., Frank, L., Paterson, M., Peroomian, V., and Zelenyi, L.M., Proton velocity distributions in the magnetotail: Theory and observations, J. Geophys. Res., 101, A2, 2587-2598, 1996.

Bardin, B., On motions near the Lagrange equilibrium point $L_{4}$ in the case of Routh's critical mass ratio, Celestial Mechanics and Dynamical Astronomy, 82, 163-177, 2002.

Bogolyubov, N.N., Mitropolsky, Yu.A., Asymptotic Methods in the Theory of Nonlinear Oscillations, New York: Gordon and Breach Science Publ., 537, 1961.

Bruhwiler, D., and Cary, J., Diffusion of particles in a slowly modulated wave, Physica D, 40, 265-282, 1989.

Büchner, J., and Zelenyi, L.M., Regular and chaotic charged particle motion in magnetotaillike field reversals 1. Basic theory of trapped motion, J. Geophys. Res., 94, 11821-11842, 1989.

Cary, J.R., Escande D.F., and Tennyson J., Adiabatic invariant change due to separatrix crossing, Phys. Rev., A34, 4256-4275, 1986.

Cary, J.R., and Scodje R., Phase change between separatrix crossings, Physica D, 36, 287-316, 1989.

Cattell, C., Roth, I., and Linton, M., The effects of low frequency waves on ion trajectories in the Earth's magnetotail, Geophys. Res. Lett., 22, 3445-3448, 1995.

Chen, J., and Palmadesso, P.J., Chaos and nonlinear dynamics of single particle orbits in magnetotail like field magnetic field, J. Geophys. Res., 91, 1499-1508, 1986.

Chen J., Burkhardt, G.R., and Huang, C.Y., Observational signatures of nonlinear magnetotail particle dynamics, Geophys. Res. Lett., 17, 2237-2240, 1990.

Chen, J., Nonlinear dynamics of charged particles in the magnetotail, J. Geophys. Res., 97, 15011-15050, 1992. Chernikov, A.A., Schmidt, G., and Neishtadt, A.I., Unlimited particle acceleration by waves in a magnetic field, Phys. Rev. Lett., 168, 1507-1510, 1992.

Chirikov, B.V., Passage of nonlinear oscillatory system through resonance, Sov. Phys. Dokl., 4, 390-394, 1959. Elskens Y., and Escande, D., Slowly pulsating separatrices sweep homoclinic tangles where islands must be small: an extension of classical adiabatic theory, Nonlinearity 4, 615-667, 1991.

Fux, R., Order and chaos in the local disc stellar kinematics induced by the Galactic bar, Astronomy \& Astrophysics, 373, 511-535, 2001. 
Gendelman, O., Manevitch, L.I., Vakakis, A.F., and M'Closkey, R., Energy pumping in nonlinear mechanical oscillators: part I-Dynamics of the underlying Hamiltonian systems, J. of Applied Mechanics - Transactions of the ASME, 68, 34-41, 2001.

Greco, A., Zimbardo, G., Veltri, P., Taktakishvili, A.L., and Zelenyi, L.M., Non Gaussian and non local transport in the Earth's distant magnetotail. Astrophys. \& Space Sci., 277, 35-38, 2001.

Grigorenko, E.E.,Fedorov, A.O., and Zelenyi, L.M., Statistical study of transient plasma structures in magnetotail lobes and plasma sheet boundary layer: Interball-1 observations. Ann. Geophysicae, 20, 329-340, 2002.

Hoffman, F., and Teller, E., Magnetohydrodynamic shock, Phys. Rev., 80, 692-703, 1950.

Itin, A.P., Neishtadt, A.I., and Vasiliev, A.A., Captures into resonance and scattering on resonance in dynamics of a charged relativistic particle in magnetic field and electrostatic wave, Physica D, 141, 281-296, 2000.

Itin, A.P., Neishtadt, A.I., and Vasiliev, A.A., Resonant phenomena in slowly perturbed rectangular billiards, Physics Lett. A, 291, 133-138, 2001.

Karney, C.F.F., Fisch, N.J., and Reiman, A.H., Green's function for RF-driven current in a toroidal plasma, AIP Conference Proceedings, 190, 430-433, 1989.

Kevorkian, J., Model for reentry roll resonance, SIAM J. Appl. Math, 26, 638-669, 1974.

Karimabadi, H., Pritchett, P.L., and Coroniti F.V., Particle orbits in two-dimensional equilibrium models for the magnetotail. J. Geophys. Res., 95, A10, 17153-66, 1990.

Lifshitz, I.M., Slutskin A.A., and Nabutovskii V.M., Motion of charged quasiparticles in a varying inhomogeneous electro-magnetic field, Sov. Phys. JETP, 14, 669-675, 1962.

Lui, A.T.Y., Multifractal and intermittent nature of substorm-associated magnetic turbulence in the magnetotail, J. of Atmospheric \& Solar-Terrestrial Physics, 63, 1379-85, 2001.

Ma, Ch., and Summers, D., Formation of power-law energy spectra in space plasma by stochastic acceleration due to whistler mode waves, Geophys. Res. Lett., 25, 4099-4102, 1998.

Neishtadt A.I., Change of an adiabatic invariant at a separatrix, Sov. J. Plasma Phys. 12, 568-573, 1986.

Neishtadt A.I. On the change in the adiabatic invariant on crossing a separatrix in systems with two degrees of freedom, PMM USSR, 51, 586-592, 1987.

Neishtadt, A.I., Petrovichev, B.A., and Chernikov, A.A., Entrainment of particles into unbounded acceleration, Sov. J. Plasma Phys., 15, 1021-1023, 1989.

Neishtadt, A.I., Scattering by resonances, Celestial Mechanics and Dynamical Astronomy, 65, 1-20, 1997. 
Neishtadt, A.I., Sidorenko, V.V., and Treschev, D.V., Stable periodic motions in the problem on passage through a separatrix, Chaos, 7, 2-11, 1997.

Neishtadt, A.I., Vainshtein, D.L., and Vasiliev, A.A., Chaotic advection in a cubic Stokes flow, Physica D, 111, 227-242. 1998.

Neishtadt, A.I., On adiabatic invariance in two-frequency systems, In: Hamiltonian systems with 3 or more degrees of freedom, NATO ASI Series C, Volume 533, p. 193-213, Kluwer Academic Publishers, 1999.

Popov A.A., Thompson, J.M.T., McRobie, F.A., Chaotic energy exchange through auto-parametric resonance in cylindrical shells, J. of Sound and Vibration, 248, 395-411, 2001.

Sagdeev, R.Z., Handbook of plasma physics, North-Holland Pub. Co.; New York, N.Y., 1983.

Timofeev A.V., On the constancy of an adiabatic invariant when the nature of the motion changes, Sov. Phys. JETP 48, 656-659, 1978.

Tsyganenko, N.A., Global quantitative models of the geomagnetic field in the cislunar magnetosphere for different disturbance levels, Planetary and Space Science, 35, 1347-1358, 1987.

Vainshtein, D.L., Neishtadt, A.I., and Zelenyi, L.M., Quasi-adiabatic description of the motion of charged particles in configurations with a reversed magnetic field, Plasma Physics Reports, 21, 457-464, 1995.

Vainshtein, D.L., Neishtadt, A.I., and Zelenyi, L.M., Quasiadiabatic description of the motion of charged particles in the vicinity of the X-line, Plasma Physics Reports, 22, 1039-1045, 1996.

Vakakis, A.F., Gendelman, O., Energy pumping in nonlinear mechanical oscillators: part II-Resonance capture, J. of Applied Mechanics - Transactions of the ASME, 68, 42-48, 2001.

Vasiliev, A.A., Neishtadt, A.I., and Itin, A.P., On dynamics of four phase-coupled oscillators with close frequencies, Regular and Chaotic Dynamics, 3, 1-9, 1997.

Veltri, P., Zimbardo, G., Taktakishvili, A.L., and Zelenyi, L.M., Effect of magnetic turbulence on the ion dynamics in the distant magnetotail. J. Geophys. Res., 103, A7, 14897-14910, 1998.

Ward, T., Homsy, G.M., Electrohydrodynamically driven chaotic mixing in a translating drop, Physics of Fluids, $13,3521-3525,2001$.

Wisdom, J., A perturbative treatment of motion near the 3/1 commensurability, Icarus, 63, 2, 272-289, 1985.

Zaslavskii, G.M., Neishtadt, A.I., Petrovichev, B.A., and Sagdeev, R.Z., Mechanism for enhanced diffusion in a wave-particle interaction in a weak magnetic field, Sov. J. Plasma Phys., 15, 638-641, 1989.

Zelenyi, L.M., Zogin, D.V., and Büchner, J., Quasiadiabatic dynamics of charged particles in the tail of the magnetosphere, Cosmic Research, 28, 369-381, 1990. 